\title{
1
}

\section{The Emerging Core and Metropolitan Networks}

\author{
Andrea Di Giglio, Angel Ferreiro and Marco Schiano
}

\subsection{Introduction}

\subsubsection{Chapter's Scope and Objectives}

The study of transport networks is a vast and highly multidisciplinary field in the modern telecommunication world. The beginner who starts studying this technical subject may remain astonished by the variety and complexity of network architectures and technologies that have proliferated in the last decade. Even an expert in the field may get disoriented in the huge variety of networks' functions and characteristics.

This introductory chapter is devoted to the definition of transport networks' fundamentals representing the very basic "toolbox" of any expert in the field. Furthermore, it investigates transport network architectural evolution in terms of new network services supporting emerging users' applications.

The chapter is structured as follows. Section 1.2 contains the definitions of the basic network concepts used throughout the book. Sections 1.3 and 1.4 describe the requirements and the architectural evolution roadmap of transport networks based on emerging users' applications. Finally, Section 1.5 shows the economic models and analysis techniques that enable the design and realization of economically sustainable transport services.

\subsection{General Characteristics of Transport Networks}

For more than a century, the traditional vision of telecommunication networks has been a smart combination of transmission and switching technologies. Even if transmission and switching are still the basic building blocks of any network, telecommunication networks fundamentals cover a much broader scope nowadays. This new vision is primarily due to the introduction of digital

Chapter 1, 'The Emerging Core and Metropolitan Networks', (c) 2009 Angel Ferreiro and Telecom Italia S.p.A from Core and Metro Networks, edited by A. Stavdas, 2009 
technologies paving the way to packet-based networks. In contrast to old analog networks, packet-based digital networks can be either connectionless or connection oriented, can have a control plane for the automation of some functions, can implement various resilience schemes, can perform a number of network services supporting users' applications, and so on.

The essential ideas are explained in this section as a background for the entire chapter.

\subsubsection{Circuit- and Packet-Based Network Paradigms}

Digital networks can transfer information between nodes by means of two fundamental paradigms: circuit switching or packet switching.

- In circuit-switched networks, data are organized in continuous, uninterrupted bit streams. In this mode of operation, a dedicated physical link between a couple of nodes is established. Before starting the data transfer on a specific connection, the connection itself must be "provisioned"; that is, the network switching nodes must be configured to provide the required physical link. This implies an exclusive allocation of network resources for the whole duration of the connection. Such a task is usually performed by dedicated elements belonging to the network control system; network resources are released when the connection ends.

This is the way that the plain old telephony service (POTS) has been working so far. The private reservation of network resources prevents other connections from using them while the first one is working, and may lead to inefficient network use.

- In packet-switched networks, data are organized in packets of finite length that are processed one by one in network nodes and forwarded based on the packet header information. In this network scenario, each packet exploits switching and transmission devices just for the time of its duration, and these network resources are shared by all packets. This process of packet forwarding and aggregation is called statistical multiplexing and represents the major benefit of packet-switched networks with respect to the circuit-switched networks in terms of network exploitation efficiency.

Typical examples of circuit-switching and packet-switching technologies are synchronous digital hierarchy (SDH) and Ethernet respectively.

Packet-switched networks can, in turn, work in connectionless or connection-oriented network modes.

- In the connectionless network mode, packets are forwarded hop by hop from source node to destination node according to packet header information only, and no transfer negotiation is performed in advance between the network nodes involved in the connection; that is, the source node, optionally the intermediate node(s) and the destination node.

- In the connection-oriented network mode, packet transfer from source node to destination node is performed through defined resource negotiation and reservation schemes between the network nodes; that is, it is preceded by a connection set-up phase and a connection usage phase, followed by a connection tear-down phase.

Typical examples of packet-switched connectionless and connection-oriented network protocols are Internet protocol (IP) and asynchronous transfer mode (ATM) respectively. 
The main characteristic of the connectionless network mode is that packets are routed throughout the network solely on the base of the forwarding algorithms working in each node; hence, packet routes may vary due to the network status. For instance, cable faults or traffic overloads are possible causes of traffic reroute: in the connectionless network mode, the new route of a packet connection is not planned in advance and, in general, is unpredictable.

On the contrary, in the connection-oriented network mode, the route of any connection is planned in advance and, in the case of faults, traffic is rerouted on a new path that can be determined in advance.

Since route and rerouting have strong impacts on the quality of a packet connection, the two network modes are used for different network services depending on the required quality and the related cost.

\subsubsection{Network Layering}

The functions of a telecommunication network have become increasingly complex. They include information transfer, traffic integrity and survivability aspects, and network management and performance monitoring, just to mention the main ones. To keep this growing complexity under control and to maintain a clear vision of the network structure, layered network models have been developed. According to these models, network functions are subdivided into a hierarchical structure of layers. Each layer encompasses a set of homogeneous network functions duly organized for providing defined services to the upper layer, while using the services provided by the lower layer. For example, in an Ethernet network, the physical layer provides data transmission services to the data link layer.

To define transport network architectures, it is essential to start from the description of the lowest three layers [1]: network, data link, and physical layers:

- Network layer. The main task of the network layer is to provide routing functions. It also provides fragmentation and reassembly of data at the endpoints. The most common layer 3 technology is the IP. It manages the connectionless transfer of data across a router-based network.

- Data-link layer. This provides frames, synchronization, and flow control. The data link layer also performs transfer of data coming from the network layer. Typical examples of datalink layers are point-to-point protocol and Ethernet MAC (medium/media access control) (IEEE 802.1xx).

- Physical layer. The physical layer defines the transmission media used to connect devices operating at the upper layer (e.g., data link). Physical media can be, for example, copper-wire pairs, coaxial cables or, more frequently, single-mode or multimode optical fibers. The physical layer also defines modulation encoding (e.g., Manchester, 8B/10B) or topology (e.g., ring, mesh) [2]. Most common technologies implementing layer 1 functionalities are Ethernet (physical layer, IEEE 802.3xx), SDH and optical transport network (OTN).

It is commonly agreed that the Open System Interconnection (OSI) model is an excellent place to begin the study of network architecture. Nevertheless, the network technologies commercially available do not map exactly with the levels described in the OSI basic model. 


\subsubsection{Data Plane, Control Plane, Management Plane}

The layered network models encompass all network functions related to data transfer. However, modern transport networks are often provided with additional functions devoted to network management and automatic network control. Hence, the totality of network functions can be classified into three groups named planes: the data plane, the management plane and the control plane.

The functions that characterize each plane are summarized below.

- Data plane. The data plane aims at framing and carrying out the physical transportation of data blocks to the final destination. This operation includes all transmission and switching functions.

- Control plane. The control plane performs the basic functions of signaling, routing and resource discovery. These are essential operations to introduce automation on high level network functions such as: connection establishment (i.e., path computation, resource availability verification and connection signaling set-up and tear-down), reconfiguration of signaled connections and connection restoration in case of network faults.

- Management plane. The management plane performs management functions like alarm reporting, systems configuration and connection provisioning for data and control planes. The complexity of the management plane depends strongly on the availability of a control plane. For example, the management plane of traditional circuit-switched public switched telephone networks is more cumbersome than transport networks with a control plane, since, in the latter case, certain tasks (e.g., connection provisioning and restoration) are carried out by the control plane itself.

\subsubsection{Users' Applications and Network Services}

The current challenge of evolving telephony-dedicated transport networks towards enhanced communication architectures is set by two fundamental trends.

First, services offered today to final users are much richer than simple telephony. User services like video telephony, video on demand, and Web browsing require an advanced terminal, typically a personal computer with dedicated software; for this reason, they will be called "user applications" or simply "applications" from now on.

Second, to convey these end-user applications, transport networks are relying on "network services," which effectively refer to a number of transfer modes.

As an example, a point-to-point unprotected circuit connection at $2 \mathrm{Mbit} / \mathrm{s}$ represents a specific transfer mode. Other examples of network services are connections based on packet paradigms; for example, IP/multi-protocol label switching (MPLS), ATM or Ethernet. Today, all modern applications make reference to packet-based network services.

The idea of a transport network able to provide many different services is one of the most challenging of recent years and it will be analyzed in detail in the following chapters.

Network services and user applications can be provided by different actors. Network operators that own and manage the networks are typical providers of network services. Service providers sell and support user applications by means of network services supplied by network operators. 
Important user application categories are:

- multimedia triple play - voice, video and high-speed Internet;

- data storage for disaster recovery and business continuity;

- grid computing; that is, computing services delivered from distributed computer networks.

The last two categories, storage and grid computing, are dedicated to business company customers and research institutions. On the contrary, multimedia applications address residential customers and the small office, home office.

Examples of network services are:

- time-division multiplexing (TDM) connections and wavelength connections (e.g., leased lines);

- Ethernet point-to-point, point-to-multipoint (p2mp) or rooted multipoint connections;

- virtual private networks (Section 1.2.8).

Each user application is enabled by a network service characterized by specific attributes. A list of the most important ones is shown below.

- Protocols: Ethernet and IP are the most common.

- Bandwidth: committed peak, committed average bit-rate, excess peak and excess bitrate [3].

- Quality of service (QoS): regarding transport networks, this is defined by means of the maximum allowed packet loss rate (PLR), the packet latency (i.e., the packet transmission delay), and jitter (latency variation); see Section 1.2.6.

- Resilience: required connection availability (Section 1.2.5).

These service attributes are the main inputs for a network provider to design a multi-service network, in support of a number of defined applications.

\subsubsection{Resilience}

One of the most important features of transport networks is their ability to preserve live traffic even when faults occur. This feature is generally referred to as "resilience."

In transport networks, resilience is usually achieved by duplication of network resources. For example, a fiber-optic link between a couple of nodes can be duplicated to assure survivability to cable breaks. Similarly, the switching matrix of an exchange node can be duplicated to guarantee service continuity in the case of electronics faults.

The way these extra resources are used depends strongly on network topology (rings or meshed network configurations), equipment technology (packet or circuit switching, network mode, optical transmission), and traffic protection requirements. However, the following general definitions help understanding the fundamental resilience schemes. 
1. If the connections for traffic protection are organized in advance, the resilience mechanism is called "protection."

a. 1+1 protection (also called dedicated protection). The whole traffic of a connection is duplicated and transmitted through two disjoint paths: the working and the protection path simultaneously. The receiving node switches between the two signals in the case of failure. The trigger of $1+1$ protection is the received signal quality; for example, the received power level or the bit error rate (BER). Since no complex network protocols are needed, $1+1$ protection works very quickly, typically within $50 \mathrm{~ms}$. The drawback of this protection scheme is duplication of network resources.

b. 1: 1 protection (also called protection with extra traffic). The working connection is protected with one backup connection using a disjoint path. The working traffic is sent over only one of the connections at a time; this is in contrast to dedicated protection, where traffic is always bridged onto two connections simultaneously. Under normal conditions (no network failure) the protecting connection is either idle or is carrying some extra traffic (typically best-effort traffic). Configuring 1: 1 protection depends on the control plane's ability to handle extra traffic, that is, whether it supports the preemption of network resources for allocating them to the working traffic once it has been affected by the failure. The ingress node then feeds the working traffic on the protecting connection in the case of failure. The trigger of 1: 1 protection is the reception of network failure notification messages. Protection with extra traffic has two main drawbacks: the need to duplicate working traffic resources onto the protection path and, in the case of resource contention, the possibility that extra traffic may be interrupted without effective need.

c. $M: N$ protection (also called shared protection). $M$ working connections are protected by $N$ backup connections on a disjoint path $(N \leq M)$. The traffic is no longer duplicated because backup connections can carry traffic initially transported by any one of the working connections in the case of fault. Thus, switching to backup connections requires first knowing their availability and then performing traffic switching. Signaling is needed for failure notification and backup connection activation. Once failure has been repaired, traffic is reassigned to the working connection and the resources of the backup connection are available again for protection. In any case, this protection mechanism allows resource savings with respect to $1+1$ protection.

Both protection mechanisms, dedicated and shared, are used in rings and meshed network configurations. The main advantage of protection is its quick operation, since the backup path is predefined and network resources are pre-allocated.

2. Alternatively to protection, restoration is the resilience mechanism that sets up new backup connections after failure events by discovering, routing, and setting up new links "on the fly" among the network resources still available after the failure. This is achieved by the extension of signaling, routing, and discovery paradigms typical of IP networks. In fact, to restore a connection, switching nodes need to discover the network topology not affected by the failure, thus allowing one to compute a set of candidate routes, then to select a new route, and to set up the backup connections. Discovery, routing algorithms, and signaling functions embedded in commercial IP/MPLS routers can quite easily implement restoration. On the other hand, transport network equipment needs a dedicated control plane to perform such functions. 
Table 1.1 Indicative figures for network availability

\begin{tabular}{llc}
\hline Availability $(\%)$ & $N$-Nines & Downtime time (minutes/year) \\
\hline 99 & 2-Nines & 5000 \\
99.9 & 3-Nines & 500 \\
99.99 & 4-Nines & 50 \\
99.999 & 5-Nines & 5 \\
99.9999 & 6-Nines & 0.5 \\
\hline
\end{tabular}

Usually, the resilience level of a network service (e.g., a leased line or an Ethernet connection, as defined in Section 1.2.4) is made precise through a number of parameters; the most important are:

- Mean time to failure (MTTF): the reciprocal of the failure rate, for systems being replaced after a failure.

- Mean time to repair (MTTR): this depends on the repair time of a network fault.

- Mean time between failures (MTBF): this is the sum of MTTF and MTTR and defines the mean time interval between successive failures of a repairable system; it is a measure of network component reliability.

- Maximum recovery time: this is the maximum delay between a failure injuring a network service and the restoration of the service over another path; in other words, the maximum time during which the network service is not available. It accounts for MTTR and all other possible delays affecting complete system recovery (signaling, rerouting).

The same concept can be given a different flavor, insisting on network status instead of duration:

- Unavailability: the probability that the network service is not working at a given time and under specified conditions; it is the ratio MTTR/MTBF. Some indicative numbers for network availability are illustrated in Table 1.1 .

\subsubsection{Quality of Service}

Network services are characterized by a set of parameters that define their quality (QoS).

- BER: this is a physical-layer parameter, manifesting the fraction of erroneous bits over the total number of transmitted bits. It is closely related to design rules applied to the physical layer transport network. It is studied in detail in Chapter 3.

- PLR: in packet-switched services, this is the fraction of data packets lost out of the total number of transmitted packets. Packets can be dropped due to congestion, or due to transmission errors or faults.

- Latency: the time needed for carrying data from the source node to the destination node. Latency is caused by the combination of signal propagation delay, data processing delays, and queuing delays at the intermediate nodes on the connection [3].

- Latency variation: the range of variation of the latency mainly due to variable queuing delays in network nodes or due to data segmentation and routing of data blocks, via different physical paths (a feature readily available in next-generation (NG)-synchronous optical network (SONET)/SDH). Also, queuing delay variations may occur in the case of traffic 
overload in nodes or links. An excess of latency variation can cause quality degradation in some real-time or interactive applications such as voice over IP (VoIP) and video over IP (IP television (IPTV)).

- Service unavailability: this has already been defined in Section 1.2.5.

For connection-oriented network services, the definition of QoS also includes:

- Blocking probability: the ratio between blocking events (failure of a network to establish a connection requested by the user, because of lack of resources) and the number of attempts.

- Set-up time: delay between the user application request time and the network service actual delivery time.

Current packet-based networks are designed to satisfy the appropriate level of QoS for different network services. Table 1.2 shows suitable values of QoS parameters for the main users' applications. As an example, applications like voice or videoconference need tight values of latency and latency variation. Video distribution is more tolerant to latency variation, but it needs low packet loss, since lost packets are not retransmitted. File transfer (e.g., backup) does not have strong requirements about any QoS parameters, since the only requirement is to transfer a pre-established amount of data in a fixed time interval.

\subsubsection{Traffic Engineering}

In complex meshed networks, careful traffic engineering (TE) and resource optimization is a mandatory requirement providing network management and operation functions at reasonable capital expenditure (CAPEX) and operational expenditure (OPEX). Towards this end, the use of conventional algorithms to set up the working and protection (backup) paths and for traffic routing within the network is insufficient. To address this problem, use is made of TE, which is a network engineering mechanism allowing for network performance optimization by means of leveraging traffic allocation in conjunction with the available network resources.

The purpose of TE is to optimize the use of network resources and facilitate reliable network operations. The latter aspect is pursued with mechanisms enhancing network integrity and by embracing policies supporting network survivability. The overall operation leads to the minimizations of network vulnerability, service outages due to errors, and congestions and failures occurring during daily network operations. TE makes it possible to transport traffic via reliable network resources, minimizing the risk of losing any fraction of this traffic.

TE leverages on some instruments that are independent of the network layer and technology:

- A set of policies, objectives, and requirements (which may be context dependent) for network performance evaluation and performance optimization.

- A collection of mechanisms and tools for measuring, characterizing, modeling, and efficiently handling the traffic. These tools allow the allocation and control of network resources where these are needed and/or the allocation of traffic chunks to the appropriate resources.

- A set of administrative control parameters, necessary to manage the connections for reactive reconfigurations. 
Table 1.2 QoS characterization of users' applications

\begin{tabular}{|c|c|c|c|c|c|}
\hline \multirow[t]{2}{*}{ User application } & \multicolumn{5}{|c|}{ QoS } \\
\hline & $\begin{array}{l}\text { Max. } \\
\text { latency } \\
(\mathrm{ms})\end{array}$ & $\begin{array}{c}\text { Max. latency } \\
\text { variation (ms) }\end{array}$ & $\begin{array}{l}\text { Packet loss } \\
\text { (layer 3) } \\
\text { (\%) }\end{array}$ & $\begin{array}{l}\text { Max. } \\
\text { set-up } \\
\text { time }\end{array}$ & $\begin{array}{l}\text { Min. } \\
\text { availability } \\
\quad(\%)\end{array}$ \\
\hline \multicolumn{6}{|l|}{ Storage } \\
\hline Backup/restore & N.A. & N.A. & 0.1 & $\min$ & 99.990 \\
\hline Storage on demand & 10 & 1 & 0.1 & $\mathrm{~s}$ & 99.999 \\
\hline Asyncrhonous mirroring & 100 & 10 & 0.1 & $\mathrm{~s}$ & 99.999 \\
\hline Synchronous mirroring & 3 & 1 & & $\min$ & 99.999 \\
\hline \multicolumn{6}{|l|}{ Grid computing } \\
\hline Compute grid & 100 & 20 & 0.0 & $\mathrm{~s}$ & 99.990 \\
\hline Data grid & 500 & 100 & 0.1 & $\mathrm{~s}$ & 99.990 \\
\hline Utility grid & 200 & 50 & 0.0 & $\mathrm{~s}$ & 99.999 \\
\hline \multicolumn{6}{|l|}{ Multimedia } \\
\hline $\begin{array}{l}\text { Video on demand (enter- } \\
\text { tainment quality, similar to } \\
\text { DVD) }\end{array}$ & $2-20 \mathrm{~s}$ & 50 & 0.5 & $\mathrm{~s}$ & $99.500 \%$ \\
\hline $\begin{array}{l}\text { Video broadcast (IP-TV), } \\
\text { entertainment quality } \\
\text { similar to DVD }\end{array}$ & $2-20 \mathrm{~s}$ & 50 & 0.5 & $\mathrm{~s}$ & 99.500 \\
\hline Video download & $2-20 \mathrm{~s}$ & 1000 & 1.0 & $\mathrm{~s}$ & 99.990 \\
\hline $\begin{array}{l}\text { Video chat (SIF quality, no } \\
\text { real-time coding penalty) }\end{array}$ & 400 & 10 & 5.0 & $\mathrm{~s}$ & 99.500 \\
\hline $\begin{array}{l}\text { Narrowband voice, data } \\
(\text { VoIP, ...) }\end{array}$ & $100-400$ & 10 & 0.5 & $\mathrm{~ms}$ & 99.999 \\
\hline Telemedicine (diagnostic) & $40-250$ & $5-40$ & 0.5 & $\mathrm{~ms}$ & 99.999 \\
\hline Gaming & $50-75$ & 10 & 5.0 & $\mathrm{~s}$ & 99.500 \\
\hline $\begin{array}{l}\text { Digital distribution, digital } \\
\text { cinema }\end{array}$ & 120 & 80 & 0.5 & $\mathrm{~s}$ & 99.990 \\
\hline $\begin{array}{l}\text { Video conference (PAL } \\
\text { broadcast quality } 2.0 \\
\text { real-time coding penalty) }\end{array}$ & 100 & 10 & 0.5 & & 99.990 \\
\hline
\end{tabular}

Note: latency is expressed in milliseconds with the exception of video on demand, video broadcast, and video download, where seconds are the unit.

The process of TE can be divided into four phases that may be applied both in core and in metropolitan area networks, as described by the Internet Engineering Task Force (IETF) in RFC 2702 [4]:

- Definition of a relevant control policy that governs network operations (depending on many factors like business model, network cost structure, operating constraints, etc.).

- Monitoring mechanism, involving the acquisition of measurement data from the actual network.

- Evaluation and classification of network status and traffic load. The performance analysis may be either proactive (i.e., based on estimates and predictions for the traffic load, scenarios 
for the scheduling of network resources in order to prevent network disruptions like congestion) or reactive (a set of measures to be taken to handle unforeseen circumstances; e.g., in-progress congestion).

- Performance optimization of the network. The performance optimization phase involves a decision process, which selects and implements a set of actions from a set of alternatives.

\subsubsection{Virtual Private Networks}

A virtual private network (VPN) is a logical representation of the connections that makes use of a physical telecommunication infrastructure shared with other VPNs or services, but maintaining privacy through the use of tunneling protocols (Section 1.2.9) and security procedures. The idea of the VPN is to give a user the same services accessible in a totally independent network, but at much lower cost, thanks to the use of a shared infrastructure, rather than a dedicated one [5].

In fact, a common VPN application is to segregate the traffic from different user communities over the public Internet, or to separate the traffic of different service providers sharing the same physical infrastructure of a unique network provider.

VPNs are a hot topic also in the discussion within standardization bodies: different views exist on what a VPN truly is.

According to ITU-T recommendation Y.1311 [6] a VPN "provides connectivity amongst a limited and specific subset of the total set of users served by the network provider. A VPN has the appearance of a network that is dedicated specifically to the users within the subset." The restricted group of network users that can exploit the VPN services is called a closed user group.

The other standardization approach, used by the IETF, is to define a VPN's components and related functions (RFC 4026, [7]):

- Customer edge (CE) device: this is the node that provides access to the VPN service, physically located at the customer's premises.

- Provider edge (PE) device: a device (or set of devices) at the edge of the provider network that makes available the provider's view of the customer site. PEs are usually aware of the VPNs, and do maintain a VPN state.

- Provider (P) device: a device inside the provider's core network; it does not directly interface to any customer endpoint, but it can be used to provide routing for many provideroperated tunnels belonging to different customers' VPNs.

Standardization bodies specified VPNs for different network layers. For example, a transport layer based on SDH can be used to provide a layer 1 VPN [8, 9]. Layer 2, (e.g., Ethernet) allows the possibility to implement L2-VPN, also called virtual LAN (VLAN). Layer 3 VPNs are very often based on IP, and this is the first and the most common VPN concept.

In some situations, adaptation functions between the bit-stream that is provided from the "source" (of the applications) and the VPN are required. An example of an adaptation data protocol function is the mapping of Ethernet frames in NG-SDH containers. 


\subsubsection{Packet Transport Technologies}

Packet technologies have been dominating the local area network (LAN) scenario for more than 25 years, and nowadays they are widely used also in transport networks, where many network services are based on packet paradigms. The main reason for this success is twofold: first, the superior efficiency of packet networks in traffic grooming due to the statistical aggregation of packet-based traffic; second, the inherent flexibility of packet networks that can support an unlimited variety of users' applications with a few fundamental network services, as shown in Section 1.2.4.

However, until now, the transport of packet traffic has been based on the underlying circuitswitched technology already available for telephony. A typical example is represented by Ethernet transport over NG-SDH networks. This solution is justified by the widespread availability of SDH equipment in already-installed transport networks, and by the excellent operation, administration, and maintenance (OAM) features of such technology. These features are fundamental for provisioning packet network services with the quality required for most users' applications, but they are not supported by the LAN packet technologies.

This situation is changing rapidly, because a new generation of packet-based network technologies is emerging. These new scenarios combine the efficiency and flexibility of packet networks with the effective network control and management features of circuit-based networks. These new technologies are referred to as packet transport technologies packet transport technology (PTTs).

There are proposals for introducing tunnels ${ }^{1}$ facilitating to allow Ethernet attaining traffic engineering features rendering it into a connection-oriented platform. These developments are currently under standardization at IEEE and ITU-T where is known as Provider Backbone Bridge with Traffic Engineering (or simply PBB-TE).

An alternative approach under standardization at the ITU-T and IETF is to evolve the IP/MPLS protocol suites to integrate OAM functions for carrier-grade packet transport networks.

This PTT, known as MPLS-TP (MPLS transport profile) includes features traditionally associated with transport networks, such as protection switching and operation and maintenance (OAM) functions, in order to provide a common operation, control and management paradigm with other transport technologies (e.g., SDH, optical transport hierarchy (OTH), wavelength-division multiplexing (WDM)).

The trend imposed by the dramatic increase of packet traffic and the obvious advantages in evolving existing circuit-switched networks into advanced packet-switched networks is going to make PTTs a viable solution to building a unified transport infrastructure, as depicted in Figure 1.1. Incumbent network operators that have already deployed a versatile NG-SDH network for aggregated traffic may follow conservative migration guidelines for their core networks and keep circuit solutions based on optical technologies. These plausible solutions are discussed in Section 1.4.

\footnotetext{
${ }^{1}$ A tunnel is a method of communication between a couple of network nodes via a channel passing through intermediate nodes with no changes in its information content.
} 


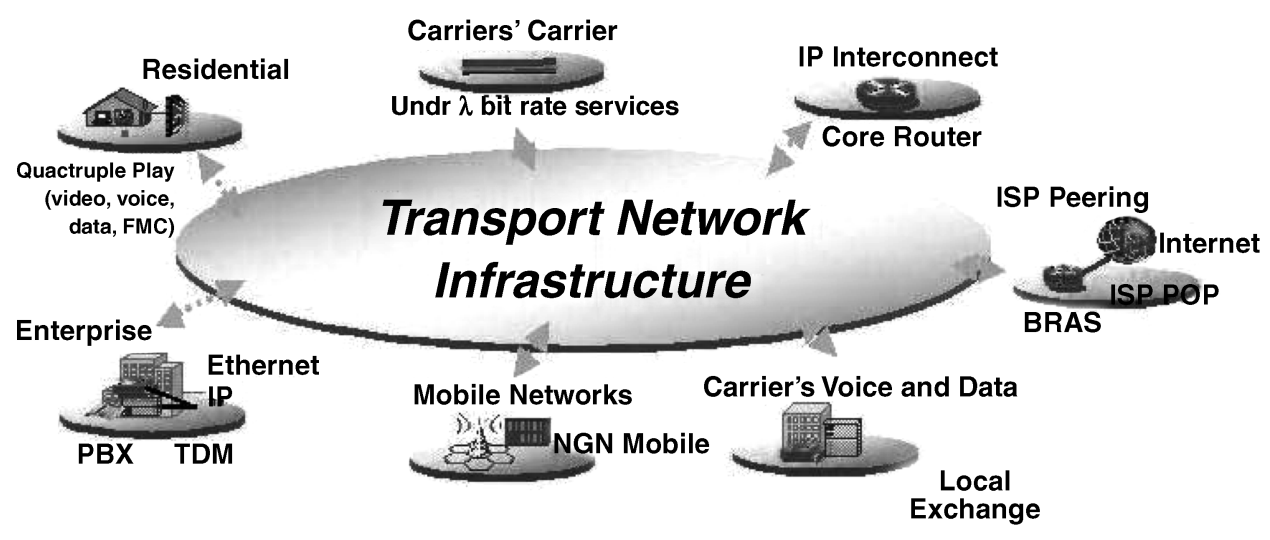

Figure 1.1 Unified transport network

\subsection{Future Networks Challenges}

\subsubsection{Network Evolution Drivers}

In the past decade, the proliferation of electronic and fiber-optic technologies has allowed network services to evolve from the exclusive support of plain telephony to an abundance of services which are transported based on the IP. These advances have had a major impact on the drivers for network evolution.

Nowadays, network design and planning is the outcome of the interplay between different technological, legal, and economic drivers:

- Introduction of new services. A network operator or a service provider can decide to offer new services based on customers' requests or market trends.

- Traffic growth. The growing penetration and the intensive use of new services increase the network load.

- Availability of new technologies. Electronic, optical, and software technologies keep on offering new advances in transmission, switching, and control of information flows based on circuits and packets.

- Degree of standardization and interoperability of new network equipment. Modern networks are very complex systems, requiring interaction of various kinds of equipment by means of dedicated protocols. Standardization and interoperability are key requirements for a proper integration of many different network elements.

- Laws and regulations. National laws and government regulations may set limitations and opportunities defining new business actors for network deployment and usage.

- Market potential and amount of investments. The financial resource availability and the potential of the telecommunication market are the key economic drivers for network development.

\subsubsection{Characteristics of Applications and Related Traffic}

In this section, the association between applications and network services is presented. The starting point of the analysis is the bandwidth requirement (traffic) of the various applications 
and the subsequent classification of this traffic into classes. Figure 1.2, illustrates a classification of user applications based on the following traffic characteristics:

- elasticity

- interactivity

- degree of resilience (availability)

- symmetry

- bandwidth.

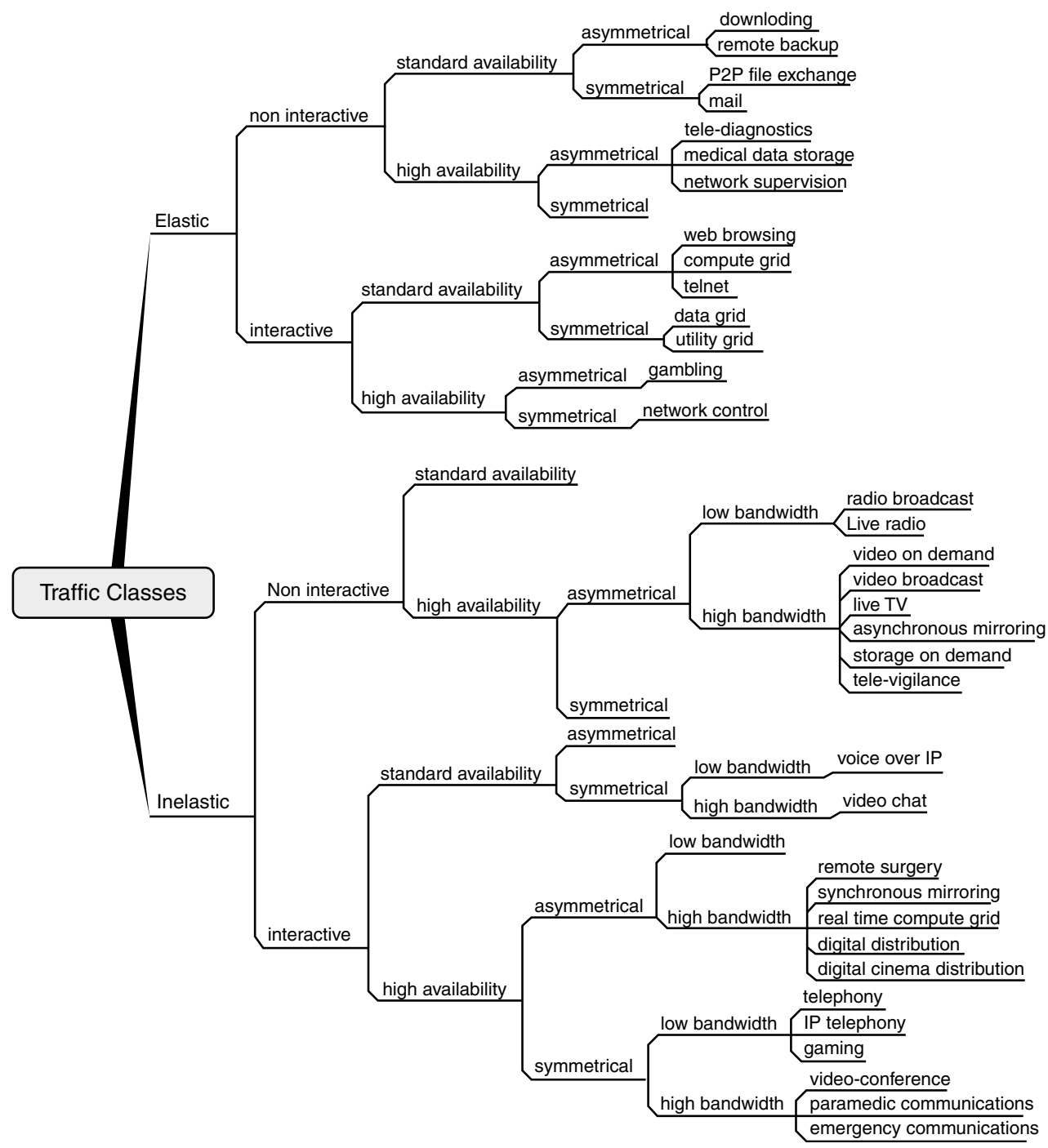

Figure 1.2 Classification of traffic generated by reference applications 
Table 1.3 Qualitative classification of traffic types

\begin{tabular}{lll}
\hline & Elastic & Inelastic \\
\hline Interactive & Transactional & Real time \\
Noninteractive & Best effort & Streaming \\
\hline
\end{tabular}

Elasticity refers to the level up to which the original traffic shape can be modified; the two main categories are as follows:

- Inelastic traffic (or stream traffic) is generated by applications whose temporal integrity overwhelms data integrity because they try to emulate virtual presence.

- Elastic traffic is generated by applications where data integrity overwhelms temporal integrity, therefore being rather tolerant to delays and being able to adapt their data generation rate to network conditions.

The term interactivity refers to a mode of operation characterized by constant feedback and an interrelated traffic exchange between the two endpoints of the connection.

To map users' applications traffic into the appropriate network services, it is essential to define a few classes of traffic patterns that share the main characteristics. For this purpose, Table 1.3 defines four kinds of traffic patterns in terms of QoS requirements.

Another important task is to assign QoS parameters quantitatively to the traffic classes. Table 1.4 sets the values of QoS parameters used to define four basic classes as:

- real-time traffic

- streaming traffic

- transactional traffic

- best-effort traffic.

In connection with Table 1.4, the term dynamicity refers to the ability of a user to modify the parameters of an existing connection. It is the only important parameter not described in Section 1.2.6, since it is not addressed directly by the classic QoS definition, but it is anyway an important quantity for application classification. The dynamicity refers to the time variation of the following connection characteristics:

- bandwidth (bit-rate);

- QoS parameters (latency, availability, data integrity);

- connectivity (the end-points of the connection).

The level of dynamicity is quantified on a three-state base:

- "none" (it is not possible to modify any parameters of an existing connection);

- "bit-rate and QoS" (when only these two parameters can be altered);

- "full" (bit-rate, QoS parameters, and connectivity modifications are allowed).

As seen in connection with Table 1.4, four traffic categories are defined based only on QoS parameters. Table 1.5 shows examples of applications belonging to each one of the four classes identified above, having different bandwidth requirements. 


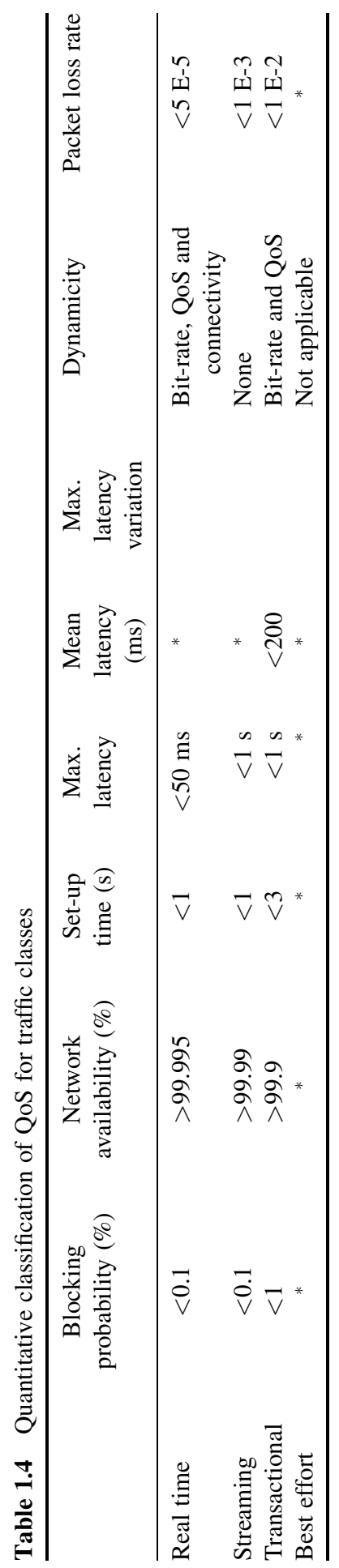


Table 1.5 Traffic characterization based on bandwidth (BW) and QoS parameters and map of users' applications

\begin{tabular}{llll}
\hline QoS & Low BW (tens of kbit/s) & Medium BW $(<2 \mathrm{Mbit} / \mathrm{s})$ & High BW $(>2 \mathrm{Mbit} / \mathrm{s})$ \\
\hline Real time & Legacy and IP telephony & Gaming & $\begin{array}{c}\text { Video conference, grid } \\
\text { computing }\end{array}$ \\
Streaming & UMTS & $\begin{array}{c}\text { Remote backup, network } \\
\text { supervision }\end{array}$ & $\begin{array}{c}\text { TV and video broadcast, } \\
\text { VoD }^{a}\end{array}$ \\
$\begin{array}{l}\text { Transactional } \\
\text { Best effort }\end{array}$ & $\begin{array}{l}\text { E-commerce } \\
\text { E-mail, domotic, VoIP }\end{array}$ & $\begin{array}{l}\text { Telnet } \\
\text { p2p file exchange, data } \\
\text { acquisition }\end{array}$ & $\begin{array}{c}\text { p2p file exchange, data } \\
\text { acquisition }\end{array}$ \\
\hline
\end{tabular}

${ }^{a}$ Video on demand.

${ }^{b}$ Storage area network.

Table 1.5 is useful to map most common users' applications into the four traffic classes (realtime, streaming, transactional, best-effort), taking also the bandwidth use into account.

Similar to the classification of user applications, network services are classified into five categories in association with the network services reported in Section 1.2.4. Thus, the network service map looks as follows:

- L1 VPN, which provides a physical-layer service between customer sites belonging to the same closed user group. These VPN connections can be based on physical ports, optical wavelengths, or TDM timeslots.

- L2 VPN, which provides a service between customer terminals belonging to the VPN at the data link layer. Data packet forwarding is based on the information contained in the packets' data link layer headers (e.g., frame relay data link circuit identifier, ATM virtual circuit identifier/virtual path identifier, or Ethernet MAC addresses).

- L3 VPN, which provides a network layer service between customer devices belonging to the same VPN. Packets are forwarded based on the information contained in the layer 3 headers (e.g., IPv4 or IPv6 destination address).

- Public IP, which is considered as the paradigm of best-effort network services. Namely, it is a generalized L3 VPN without restrictions to the user group, but with a consequently poor QoS.

- Business IP, which is included as a higher priority class that, for instance, can efficiently handle latency ${ }^{2}$ in time-sensitive applications.

On top of this classification, further "orthogonal" categorizations are often introduced. VPN services are further subdivided into:

- permanent VPNs, to be provided on a permanent basis by the network service provider;

- on-demand VPNs, which could be controlled dynamically by the client user/network.

\footnotetext{
${ }^{2}$ See latency and other $\mathrm{QoS}$ defining parameters later in this section.
} 
Table 1.6 Mapping network services groups to some applications (BW: bandwidth)

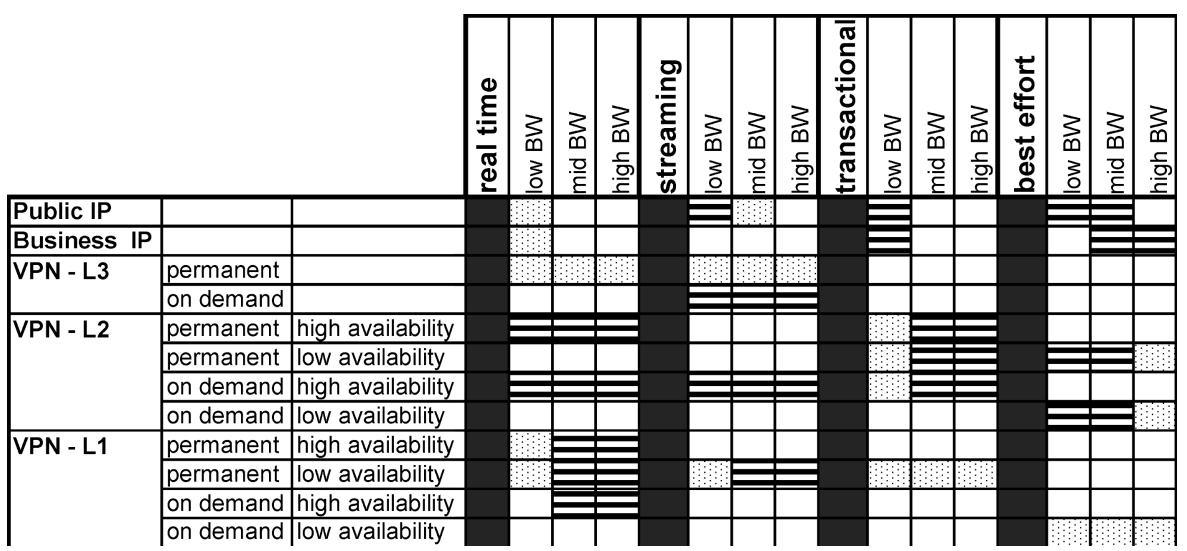

L1 and L2 VPN services are also classified into high- and low-availability services. Table 1.6 provides a mapping between "user applications" and "network services": in this context, a stippled box means that that particular application may run over on this network service, but not very efficiently. The most efficient support to that application is designated with horizontal rows, whereas a white box should be interpreted as no support at all from this service to that application.

\subsubsection{Network Architectural Requirements}

This section gives an overview of the architectural requirements for transport networks supporting the services described above.

\subsubsection{Network Functional Requirements}

From an architectural point of view, data services have been traditionally transported over a wide set of protocols and technologies. For example, IP services are transported over the core network usually relying on SDH, ATM, or Ethernet transmission networks. A widespread alternative used in current convergent transport networks is to go towards a meshed network of IP/MPLS routers, interconnected through direct fiber or lambda connections and without any multilayer interaction.

This "IP-based for everything" approach was proved to be valid for the last decade, but with current traffic trends it would lead to scalability problems. Currently, backbone nodes need switching capacities of several terabits per second, and this need is predicted to double every 2 years. Routers are also very expensive and they are not optimized for high-bandwidth traffic transportation, while transport technologies such as SONET/SDH are not efficient enough for packet transport, due to a very coarse and not flexible bandwidth granularity. 
On the other hand, a number of emerging services (e.g., new multimedia applications served over the Internet; i.e., real-time high-bandwidth video services) are imposing new requirements on the current "IP-based for everything" architecture in terms of bandwidth and QoS (end-to-end delay and availability). Moreover, mobility of users and devices and new traffic profiles (due to, for example, flash crowds and streaming services) require a network with an unprecedented dynamicity that is able to support unpredictable traffic patterns.

\subsubsection{Network Scalability}

The term scalability is a feature of a network architecture designating the ability to accommodate higher traffic load without requiring large-scale redesign and/or major deployment of resources. A typical (negative) example manifesting lack of scalability is an SDH ring where additional resources and manual configurations are mandatory in order to increase the capacity between two nodes. Thus, future transport networks should be scalable in order to support existing or yet-unknown clients and traffic volumes.

The lack of scalability is demonstrated in two distinctive ways. First, by means of an excessive deployment of network resources to accommodate higher traffic volumes. This inefficiency is leading to higher CAPEX and OPEX that are mainly attributed to the enduring very high cost of switching. Solving this issue requires the deployment of technologies able to transport traffic with a lower cost per bit. Second, it is associated with architectural and/or control plane scalability restrictions due to the excessive number of network elements to control (e.g., the number of paths in the network). To address this issue requires the adoption of layered architectures and aggregation hierarchies.

\subsubsection{Network Reconfiguration Ability}

Network reconfiguration ability refers to the ability of the network to change the status of some or all of the established connections, to modify the parameters of these connections (e.g., modify the amount of allocated bandwidth) or to modify the way the services are provided (for instance, changing the routing of a given connection to allow more efficient grooming on a different route or improve spare capacity sharing).

The interest in having a reconfigurable network comes from the fact that traffic profiles change very frequently, may be fostered by symmetrical traffic patterns, unexpected traffic growth, possible mobile data/multimedia services, varied geographic connectivity (e.g., home, work), and emerging services, such as user-generated content. All these facts make it reasonable to think in the future about a highly varying traffic profile in a network, thus meaning that reconfigurability would be a highly advantageous characteristic in data architectures.

\subsubsection{Cost Effectiveness}

Taking into account the fierce competition and the pressure upon network operators in the telecommunications market, as well as the descending cost per bit charged to the final user, the only solution for service providers to keep competitive is to reduce traffic transport costs. Therefore, cost effectiveness is the obvious requirement for any new technology. Basic 
approaches to achieve this cost reduction are to build networks upon cheap scale-economy technologies, adapted to the applications' bursty data traffic and specifically designed to keep functional complexity to a minimum. To facilitate this cost per bit reduction even in presence of unpredictable traffic growth, modular solutions are of paramount importance.

\subsubsection{Standardized Solutions}

Standardization of solutions is a key point, because it assures interoperability of equipment from different manufacturers and, as a consequence, it allows a multi-vendor environment.

This leads to the achievement of economies of scale that lower costs, since a higher number of suppliers use the same technology. Besides, standardization allows network operators to deploy networks with components from different suppliers, therefore avoiding dependence on a single manufacturer, both from a technological and an economical point of view.

\subsubsection{Quality of Service Differentiation}

As specified in Sections 1.2.6 and 1.3.2, a differentiating feature between the various applications consists in their dissimilar transport network requirements (e.g., minimum/ maximum bandwidth, availability, security, delay, jitter, loss, error rate, priority, and buffering). For this reason, networks have to support QoS differentiation because their main goal is to assure a proper multi-service delivery to different applications. The intention of QoS specifications is to utilize network mechanisms for classifying and managing network traffic or bandwidth reservation, in order to deliver predictable service levels such that service requirements can be fulfilled.

\subsubsection{Resilience Mechanisms}

As reported in Section 1.2.5, an important aspect that characterizes services offered by telecommunication networks is service availability. Resilience mechanisms must be present in order to react to network failures, providing backup solutions to restore the connections affected by the failure. Typical resilience mechanisms provide full protection against all single failures; they distinguish in terms of how fast restoration is provided and on the amount of backup capacity required for protection, to fully support this single-failure event. Resilience schemes can also be characterized depending on their ability to provide various level of protection (e.g., full protection against single failures, best effort protection, no-protection, and preemption in the case of failure) and on their capability to provide very high availability services (e.g., full protection against multiple failures). For transport network clients, the important aspect is the resulting service availability, measured in terms of average service availability over a given period of time (e.g., 1 year) and of maximum service interruption time.

\subsubsection{Operation and Maintenance}

A fundamental requirement is to keep a proper control over the networking infrastructure: easy monitoring, alarm management, and configuration tools are required. The current trend for 
OPEX reduction and maintenance simplification leads towards automated distributed control maintenance and operations.

Transport technologies or carrier-grade switching and transmission solutions differ from other technologies in the OAM features: it is important not only in administrating and managing the network, but also to provide services and to deal with its customers. Efficient operation tools and mechanisms must also be implemented within the transport networks.

Finally, it is important to consider the interoperability between different network layers that requires mutual layer independence; for this reason, the transport technology needs to be selfsufficient to provide its own OAM, independently of its client and server layers.

\subsubsection{Traffic Multicast Support}

A multicast transfer pattern allows transmission of data to multiple recipients in the network at the same time over one transmission stream to the switches.

A network with multicast capability must guarantee the communication between a single sender and multiple receivers on a network by delivering a single stream of information to multiple recipients, duplicating data only when the multiple path follows different routes. The network (not the customer devices) has to be able to duplicate data flows. There are only two degrees for the ability to support multicast transfer: able or unable (multicast is an on/off property).

Multicast distribution is considered a useful tool for transport technologies when dealing with IPTV and similar applications. However, it is pointed out that layer 2 multicasting is not the only solution to distribute IPTV.

\subsubsection{Multiplicity of Client Signals}

Previous sections highlighted that metro-core networks are supporting traffic from many different applications, such as business data, Web browsing, peer to peer, e-Business, storage networking, utility computing, and new applications such as video streaming, video conference, VoIP, and tele-medicine applications. The prevalence of multimedia services and the expansion of triple-play has an important role in traffic load and distribution in metro and core networks. A strong increase of broadband access penetration, based on a combination of different fixed and mobile access technologies, is expected for the next years, favoring the increase of terminal nomadism, which might introduce a more variable and unpredictable traffic, especially in the metro area. On the other side, corporate VPN services ranging from MPLS-based VPNs [10] to legacy services cope with the business telecom market.

From a technological standpoint, most services are migrating to packet-based Ethernet framing. This trend makes it mandatory for Core/Metro networks to support Ethernet client services. Nevertheless, many legacy networks are still based on other standards, such as SDH and ATM, and they still need to support these kinds of technology.

A transport infrastructure that can carry traffic generated by both mobile and fixed access is an important challenge for future transport networks.

Fixed and mobile applications present similar QoS requirements, and can be classified according to the four classes previously defined in Section 1.2.4. (i.e., best-effort, streaming, 
real-time, and transactional). However, current bandwidth requirements are lower for mobile applications than for fixed applications due to limitations in wireless access bandwidth and terminal screen size and resolution.

\subsubsection{Transport Network Service Models and Client Interactions}

Telecom networks have been upgraded with different network layer technologies, each providing its own set of service functionality based on its own switching paradigm and framing architecture. The GMPLS (Generalized Multi-Protocol Label Switching) protocol architecture paves the way for a convergence between transport and client networks reducing, thus, the overall control and management complexity. GMPLS can be configured to handle networks with dissimilar switching paradigms (on data plane) and different network management platforms (on control and management plane). This is made feasible by means of LSPs (Label Switched Paths) that are established between two end points. i.e. under the GMPLS protocol architecture the resources of the optical transport network are reserved based on the connectivity requests from a client packet-switched network.

\section{The Overlay Model}

The overlay model refers to a business model in which carriers or optical backbone (bandwidth) providers lease their network facilities to Internet service providers (ISPs). This model is based on a client-server relationship with well-defined network interfaces (or automatic switched optical network (ASON) reference points) between the transport network involved and client networks. The overlay model mandates a complete separation of the data client network control (that could be IP/MPLS based) and the transport network control plane (e.g., wavelength-switched optical networks/GMPLS). A controlled amount of signaling and restricted amount of routing messages may be exchanged; as a consequence, the overlay model is a very opaque paradigm. The IP/MPLS routing and signaling controllers are independent of the routing and signaling controllers within the transport domain, enabling the different networks to operate independently. The independent control planes interact through a userto-network interface (UNI), defining a client-server relationship between the IP/MPLS data network and the wavelength-switched optical network (WSON)/GMPLS transport network.

Overlay network service models support different business and administrative classes (as developed in Section 1.5.3.) and preserve confidentiality between network operators. The connection services are requested from client networks to the transport network across distinct UNIs. When a connection is established in the transport network for a given client network, this connection can be used as a nested LSP or a stitched LSP to support the requirements of the client network.

The service interface in the overlay network model can be configured according to the level of trust of the two interacting structures. The interface can be based on a mediation entity such as an operation service support (OSS) or it can use the northbound interface of the network management system. Further, the interface between client network (higher layer network) and transport network (lower layer network) can operate a GMPLS signaling protocol, such reservation protocol with TE (RSVP-TE). 


\section{Peer Model}

Compared with the overlay model, the peer model is built on a unified service representation, not restricting any control information exchanged between the transport network and the clients. This model is relevant and represents an optimal solution when a transport network operator is both an optical bandwidth provider and an ISP. In this case, the operator can optimally align the virtual topologies of its transport network with the network services required by its data network. The IP/MPLS control plane acts as a peer of the GMPLS transport network control plane, implying that a dual instance of the control plane is running over the data network (say, an IP/MPLS network) and optical network (say, a WSON/GMPLS network). The peer model entails the tightest coupling between IP/MPLS and WSON/GMPLS components. The different nodes are distinguished by their switching capabilities; for example, packet for IP routers interconnected to photonic cross-connects (PXCs).

\section{Integrated Model}

Compared with the peer model, the integrated model does not require different service interfaces between the different networks. The integrated model proposes the full convergence of data network control plane and transport network control plane. All nodes are label-switched routers (LSRs) all supporting several switching capabilities; say, wavelength, SDH and Ethernet. Each LSR is also able to handle several orders of the same switching capability as it happens; for example, with SDH. An LSR embeds one GMPLS control plane instance and is able to control simultaneously different switching capability interfaces. Only this model can handle a complete and global optimization of network resource usages through transport and client networks.

\section{Augmented Model}

The augmented model considers that the network separation offered by the overlay model provides a necessary division between the administrative domains of different network service providers, but also considers that a certain level of routing information should be exchanged between the transport network and the client networks. In a competitive environment, a complete peer network service model is not suitable because of the full exchange of topology information and network resource status between client and server optical networks, imposing on a network operator to control the resources of the client data networks and triggering the scalability issues of the management functions.

The augmented model provides an excellent support for the delivery of advanced connectivity services as might be offered from a multilayer network (MLN)/multiregion network (MRN). The capability, such as wavelength service on demand, integrated TE or optical VPN services, can require controlled sharing of routing information between client networks and the optical transport network.

\section{User-to-network Interface}

The UNI is a logical network interface (i.e., reference point) recommended in the "Requirements for Automatic Switched Transport Network" specification ITU-T G.807/Y.1302. The UNI defines the set of signaling messages that can be exchanged between a client node and a server node; for instance, an IP router and an SDH optical cross-connect (OXC) respectively. The server node provides a connection service to the client node; for example, the IP router can request TDM LSPs from its packet over SONET (PoS) interfaces. The UNI supports the exchange of authentication, authorization, and connection admission 
control messages, and provides the address space set of the reachable nodes to the client network. Different versions of the implementation agreement for a UNI have been produced by the Optical Internetworking Forum (OIF) since October 2001 as OIF UNI 1.0. The different OIF implementation agreement versions are supporting the overlay service model as well as the augmented service model. The signaling messages exchanged between the client node and the server node are focused on the LSP connection request, activation, deactivation, and tear down. The IETF specifies a GMPLS UNI that is also applicable for a peer model. Fully compliant with RSVP-TE, GMPLS UNI allows the end-to-end LSP handling from the ingress customer edge equipment to the egress customer edge equipment and at each intermediate LSR involved in the signaling sessions.

\section{Network-to-network Interface}

The network-to-network interface (NNI) is a logical network interface (i.e., reference point) recommended in the "Requirements for Automatic Switched Transport Network" specification ITU-T G.807/Y.1302. The NNI defines the set of both signaling messages and routing messages that can be exchanged between two network server nodes; for example, SONET OXC and SDH OXC. There are two types of NNI, one for intranetwork domains and one for internetwork domains: an external NNI (E-NNI) and an internal NNI (I-NNI) respectively.

- The E-NNI assumes an untrusted relationship between the two network domains. The routing information exchanged between the two nodes located at the edge of the transport

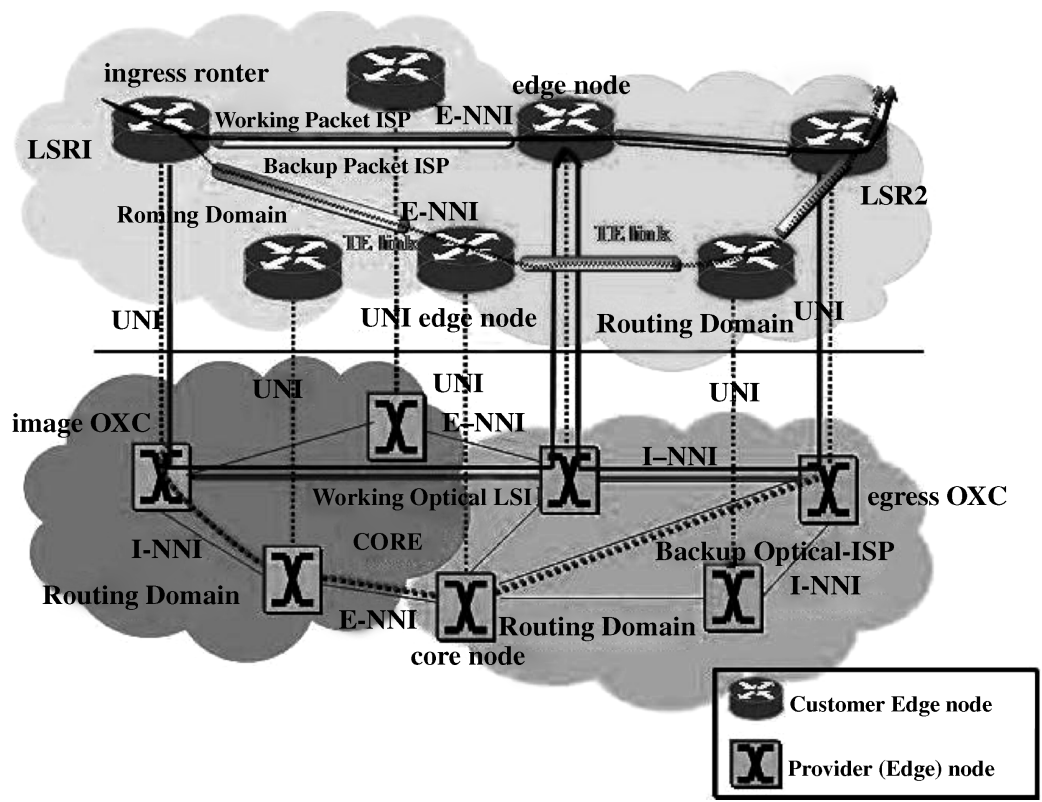

Figure 1.3 Customer nodes to public network link through server nodes by the UNI as defined in ITU architecture 
network specified within the E-NNI is restricted. The control messages exchanged include reachable network addresses that are usually translated, authentication and connection admission control messages, and a restricted set of connection requests of signaling messages.

- The I-NNI assumes a trusted relationship between two network domains. The control information specified within the I-NNI is not restricted. The routing control messages exchanged include topology, TE link state, and address discovery. The signaling messages can allow controlling of the resources end to end between several network elements and for each LSP and its protection path.

\subsubsection{Data Plane, Control Plane, and Management Plane Requirements}

\subsubsection{Data Plane Requirements}

The challenges of the physical layer part of the data plane are covered in Chapters 3-6. In this section, two conceptual challenges of the data plane are addressed, namely the quest for transparency and the search for novel transport formats.

\section{Transparency}

During the last 20 years the cornerstone of transport network evolution has been the notion of "transparency." Today, there are two distinctive understandings of the term "transparency": bit-level transparency and optical transparency.

In the original idea, the two meanings were synonymous and they were based on the following simple concept. The advent of the erbium-doped fiber amplifier facilitated the proliferation of WDM (these topics are discussed in Chapters 3-6), which increased the product "bandwidth times length" to about two to three orders of magnitude, an event that eventually led to a significant curb of the transmission cost. As a result of this evolution, the cost of switching started dominating (and still does) the cost of a transport network. At the same time, there was a clear disparity between the data throughput that the fiber-optic systems could transmit and the amount of data that synchronous systems (SDH/SONET) could process, a phenomenon that was termed an "optoelectronic bottleneck." For these reasons, every effort was made to minimize electronic switching wherever possible, making use of opticalbypassing concepts for the transit traffic, namely avoiding transport schemes requiring frequent aggregation and grooming of the client signals through electronic switches.

The widespread deployment of applications based on the IP and the emergence of a "zoo" of other protocols (like Ethernet, ESCON, Fiber Channel, etc.) gave a renewed impetus to incentivize transparency in transport networks.

In more recent times, transparency went colored of different shades.

\section{Bit-level Transparency}

The transport network (OTN in particular) should convey client signals with no processing of the information content. This will minimize the aggregation/grooming used throughout the network, whilst it will provide a client/provider agnostic transportation (transparent bitmapping into the transport frame). Here, transparency mainly indicates service transparency; that is, the minimization of bit-by-bit processing regardless of the technological platform that is used. This definition is shifting the interest from technologies to functions; hence, both 
all-optical and optoelectronic subsystems are of equal interest in building service-transparent networks.

\section{Optical Transparency}

Nevertheless, in conjunction with bit-level transparency, the initial notion of a transparent network is still of interest, where the optical/electrical/optical $(\mathrm{O} / \mathrm{E} / \mathrm{O})$ conversions are minimized so the signal stays in the optical domain. The benefits from the reduction in the number $\mathrm{O} / \mathrm{E} / \mathrm{O}$ conversions include:

- reduction of a major cost mass by minimizing the number of transponders and large (and expensive) switching machinery;

- improved network reliability with a few in number of electronic systems;

- significant reduction in power consumption (from switching fabrics to cooling requirements).

In an optically transparent network, the routing of the signal is based on the wavelength and/or on the physical port of the signal. Framing takes place at the ingress to the optically transparent domain and it adds overhead information that makes it possible to detect errors possibly occurring during transmission - at the egress node. Each standardized format has a specific frame, and several different frames are possible at the ingress to an optically transparent domain; for example, Ethernet frames, synchronous transport module frames, and G.709 [11] frames.

\section{Ethernet as an Alternative Transport Platform}

Ethernet is a frame-based technology that was defined in 1970s. It was originally designed for computer communications and for broadcasting, and since then it has been widely adopted. This was made possible thanks to two main competitive advantages. First, a successfully implemented switching capability besides the original broadcasting LAN technology. Second, because all generations of Ethernet share the same frame formats, making it feasible to support interfaces from $10 \mathrm{Mbit} / \mathrm{s}$ over copper to $100 \mathrm{Gbit} / \mathrm{s}$ over fiber (the latter still under standardization), thus ensuring seamless upgradeability.

Nowadays, Ethernet represents the most successful and widely installed LAN technology and is progressively becoming the preferred switching technology in metropolitan area networks (MANs). In the latter scenario, it is used as a pure layer 2 transport mechanism, for offering VPN services, or as broadband technology for delivering new services to residential and business users. Today, Ethernet traffic is rapidly growing and apparently it has surpassed SDH traffic. As mentioned in the previous section, bit transparent mapping is essential for a cost-effective transportation of data and it is a feature provided by Ethernet thanks to its framing format. As Ethernet is becoming the dominant technology for service provider networks and as 40/100 GbE interfaces will be standardized in the years to come, it is essential to keep Ethernet transport attractive and simple. Currently, under the Ethernet umbrella, three network layers are considered:

- Network layer, based on Metro Ethernet Forum (MEF) documents. The network services include E-Line, defining point-to-point connections, and E-LAN, defining multipointto-multipoint connections and rooted multipoint connections. 
- Layer 2, which is also called the MAC layer. This provides network architectures, frame format, addressing mechanisms, and link security (based on IEEE 802.1 and IEEE 802.3).

- Physical layer, which includes the transmission medium (e.g., coaxial cable, optical fiber), the modulation format, and network basic topologies (based on IEEE 802.3).

The numerous networks where Ethernet is installed confirm that it is a valuable frame-based technology, capable of assuring an inexpensive physical stratum, providing high bit-rates, and allowing network architectures to offer emerging network services for distributing both pointto-point and $\mathrm{p} 2 \mathrm{mp}$ variable bit-rate traffic efficiently.

There are several initiatives at standardization bodies that aim at a revision of Ethernet to make it valuable and deployable in transport networks. The work carried out at IEEE, IETF, and ITU-T is improving Ethernet with faster and more efficient resilience mechanisms and valuable OAM tools for fault localization and measurement of quality parameters to verify customers' service level agreements (SLAs).

Given this success in the access area and MAN and the simplicity and transparency it offers, Ethernet is stepping forward to the core network segment under the definition of carrier Ethernet which is investigated by the MEF as "a ubiquitous, standardized, carrier-class Service and Network." Carrier Ethernet improves standard Ethernet technology facing scalability issues (assuring a granular bandwidth increment from $1 \mathrm{Mbit} / \mathrm{s}$ to $10 \mathrm{Gbit} / \mathrm{s}$ ). It also assures hard QoS mechanisms (allowing the transport on the same lambda of different traffic categories) and reliability (the network is able to detect and recover from failures with minimum impact on users). Carrier Ethernet aims to achieve the same level of quality, robustness, and OAM functions typical of circuit technologies (think of SDH or OTN) while retaining the Ethernet advantage in offering a cost-effective statistical aggregation.

\subsubsection{Control Plane Requirements}

The control plane is studied in detail in Chapter. Here, some important issues are highlighted.

\section{Provisioning of End-to-end Connections over the Entire Network}

The main function of the control plane is to set up, tear down, and maintain an end-to-end connection, on a hop-by-hop basis, between any two end-points. The applications supported from the transport network have specific QoS requirements (Section 1.2.6), which the control plane must uphold.

\section{Unified Control Plane}

In the quest to upgrade or build new integrated network infrastructures, a paradigm shift has been witnessed in network design principles. The focus has shifted from a layered-network model involving the management of network elements individually at each layer, to one of an integrated infrastructure able to provide a seamless management of packets, circuits, and light paths. The reasons for this industry trend towards a unified set of mechanisms (the unified control plane), enabling service providers to manage separate network elements in a uniform way, can be traced to the historical evolution of transport and packet networks. The IP became the uncontested platform for supporting all types of application and the associated, IP-based, GMPLS provides a single, unified control plane for multiple switching layers [12]. 


\section{Horizontal Integration (Unified Inter-domain Control)}

This issue refers to the way of multidomain interconnection at control plane level. Horizontal integration refers to the situation where, in the data plane, there is at least one common switching facility between the domains, whilst the control plane topology extends over several domains. For instance, the control plane interconnection between lambda-switching-capable areas defines a horizontal integration.

\section{Control Plane and Management Plane Robustness}

In the emerging optical network architectures, the interplay between the control plane and management plane is essential to ensure fast network reconfiguration, while maintaining the existing features of SDH/SONET like robustness against failures, which is essential for the preservation of traffic continuity.

\section{Network Autodiscovery and Control Plane Resilience}

Automated network discovery refers to the ability of the network to discover autonomously the entrance of new equipment or any changes to the status of existing equipment. This task is assigned to the control plane. Additional functions of the control plane are the automated assessment of link and network load and path computation process needed to substantially reduce the service provision time and the changes invoked in the network infrastructure to support these services. Moreover, the automation is essential to reallocate resources: as customers cancel, disconnect, or change orders, the network resources can be readily made available to other customers.

The term control plane resilience refers to the ability of the control plane to discover the existing cross-connect topology and port mapping after recovering from a failure of itself. For example, when only control plane failures occur within one network element, the optical cross-connects will still be in place, carrying data traffic. After recovery of the control plane, the network element should automatically assess the data plane (i.e., optical crossconnects), and reconfigure its control plane so that it can synchronize with other control plane entities.

\section{Appropriate Network Visibility among Different Administrative Domains Belonging to Different Operators}

Administrative domains may have multiple points of interconnections. All relevant interface functions, such as routing, information exchanges about reachable nodes, and interconnection topology discovery, must be recognized at the interfaces between those domains. According to ASON policy, the control plane should provide the reference points to establish appropriate visibility among different administrative domains.

\section{Fast Provisioning}

As part of the reliable optical network design, fast provisioning of optical network connections contributes to efficient service delivery and OPEX reduction, and helps reaching new customers with broadband services.

\section{Automatic Provisioning}

To achieve greater efficiencies, optical service providers must streamline their operations by reducing the number of people required to deliver these services, and reducing the time required to activate and to troubleshoot network problems. To accomplish these objectives, 
providers are focusing on automated provisioning through a distributed control plane, which is designed to enable multi-vendor and multilayer provisioning in an automated way. Therefore, requests for services in the data network that may require connectivity or reconfiguration at the optical layer can happen in a more automated fashion. In addition, instead of provisioning on a site-by-site basis, the control plane creates a homogeneous network where provisioning is performed network-wide.

\section{Towards Bandwidth On-demand Services}

Providers can also set up services where the network dynamically and automatically increases/ decreases bandwidth as traffic volumes/patterns change. If the demand for bandwidth increases unexpectedly, then additional bandwidth can be dynamically provisioned for that connection. This includes overflow bandwidth or bandwidth over the stated contract amount. Triggering parameters for the change may be utilization thresholds, time of day, day of month, perapplication volumes, and so on.

Bandwidth on demand (BoD) provides connectivity between two access points in a nonpreplanned, fast, and automatic way using signaling. This also means dynamic reconfiguring of the data-carrying capacity within the network; restoration is also considered here to be a bandwidth on-demand service.

\section{Flexibility: Reconfigurable Transport/Optical Layer}

A network operator may have many reasons for wanting to reconfigure the network, primarily motivated by who is paying for what. Flexibility of the transport layers means a fair allocation of bandwidth between competing routes dealing with bursts of activity over many timescales. Reconfigurability increases network flexibility and responsiveness to dynamic traffic demands/ changes.

\subsubsection{Interoperability and Interworking Requirements}

\section{Multidomain Interoperability}

In many of today's complex networks, it is impossible to engineer end-to-end efficiencies in a multidomain environment, provision services quickly, or provide services based on real-time traffic patterns without the ability to manage the interactions between the IP-layer functionality of packet networks and that of the optical layer. According to proponents of ASON/ GMPLS, an optical control plane is the most advanced and far-reaching means to control these interactions.

Another important issue is that of translating resilience classes from one domain to another. The ASON reference points UNI and I-NNI/E-NNI are abstracted functional interfaces that can resolve that topic by partitioning the transport network into sub-networks and defining accurately the exchanges of control information between these partitions. As recommended in Ref. [13], the UNI is positioned at the edge of the transport network as a signaling interface used by the customer edge nodes to request end-to-end connection services between client networks, with the explicit level of availability. Routing and signaling messages exchanged at the I-NNI concern only the establishment of connections within a network domain or across the subnetwork. The E-NNI is placed between network domains or sub-networks to carry the control message exchanges between these regions of different administration. 


\section{Multi-vendor Interoperability}

The multi-vendor interoperability of metro and core solutions maximizes carrier performance and ensures the interoperability of legacy with emerging network architectures. One of the most important objectives of the development of a standardized ASON/GMPLS control plane is to contribute to interoperability, which validates the speed and ease of provisioning enabled by ASON/GMPLS in a live, multi-vendor network.

\section{Seamless Boundary in between Networks}

Given the vast amount of legacy SONET/SDH equipment, there is a clear need for an efficient interworking between traditional circuit-oriented networks and IP networks based on the packet-switching paradigm. For example, efficient control plane interworking between IP/MPLS and SONET/SDH GMPLS layers is indispensable and requires the specification of their coordination.

\subsubsection{Management Plane Requirements}

\section{Easy-to-use Network}

Emerging standards and technologies for optical networks allow for a significantly simplified architecture, easy and quick provision of services, more effective management, better interoperability and integration, and overall lower cost. In addition, it will be possible to provision services on these future networks such that global applications will be much more location independent.

\section{Transparent for Applications: Hide Network Technology to Users}

There are multiple separate service, technology, and technical considerations for networks depending on location, at the metro edge, metro core, aggregation points, long haul, and ultralong haul. Next-generation optical networking has the potential to reduce significantly or eliminate all of these barriers, especially with regard to application and end users.

To some degree, one of the key goals in this development is to create network services with a high degree of transparency; that is, allow network technical elements to become "invisible" while providing precise levels of required resources to applications and services. To allow an optimal use of the optical network infrastructure interconnecting different types of application, network service management functions are required to establish automatically connection services with adequate amount of allocated network resources. The network service management layer can rely on the routing and signaling control functions.

\section{Monitoring of End-to-end Quality of Service and Quality of Resilience}

The requirement of integrated monitoring of the (optical) performance of connections, QoS, and fault management speeds up system installation and wavelength turn-up and simplifies ongoing maintenance. Furthermore, the management plane should be able to monitor endto-end quality of resilience. That means the end-to-end type of transport plane resilience parameters (such as recovery time, unavailability, etc.) should be monitored and adhered according to the SLAs).

\section{Connectivity and Network Performance Supervision}

As networks run faster and become more complex, infrastructure, links, and devices must operate to precise levels in a tighter performance. As a result, a huge number of network 
problems stem from simple wiring and connection issues. Connectivity and performance supervision is at the heart of an efficient network management.

\section{Network Monitoring}

A monitoring system is dedicated to the supervision of the physical and optical layers of a network. Optical-layer monitoring should provide valuable, accurate information about the deterioration or drift with slow and small signal variations, helping to detect problems before they may become so serious to affect the QoS. It helps maintain the system from a lower layer's perspective.

\section{Policy-based Management (Network and Local-basis)}

Today's optical network architectures lack the proper control mechanisms that would interact with the management layer to provide fast reconfiguration. The problem of accurate intradomain provisioning in an automated manner allows satisfying the contracts with customers while optimizing the use of network resources. It is required that a policy-based management system dynamically guides the behavior of such an automated provisioning through the control plane in order to be able to meet high-level business objectives. Therefore, the emerging policybased management paradigm is the adequate means to achieve this requirement.

\section{End-to-end Traffic Management (Connection Admission Control, Bandwidth Management, Policing)}

Traffic management features are designed to minimize congestion while maximizing the efficiency of traffic. Applications have precise service requirements on throughput, maximum delay, variance of delays, loss probability and so on. The network has to guarantee the required QoS. For instance, the primary function of the connection admission control is to accept a new connection request only if its stated QoS can be maintained without influencing the QoS of already-accepted connections. Traffic management features are key elements in efficient networking.

\section{Multi-vendor Interoperability}

In the near future, network element management interfaces and OSS interfaces will be preintegrated by control plane vendors. Indeed, independent control planes increase the performance of network elements and OSS, and reduce carriers' reliance on any single network element or OSS application. This eliminates the task of integrating new network elements into a mass of OSS applications.

Connection services (respectively, connectivity services) are described from the network infrastructure operator (respectively, the service customer) point of view, which is complementary for the connections implemented through the control functions at customer edge (CE) nodes. Provider VPN services offer secure and dedicated data communications over telecom networks, through the use of standard tunneling, encryption, and authentication functions. To reconfigure automatically the provisioning of VPNs, automated OSS functions are required to enhance existing network infrastructures for supporting networked applications sharing the optical infrastructures.

Network service functions can automatically trigger addition, deletion, move, and/or change of access among user sites. The description of each connection service includes the UNI corresponding to the reference point between the provider edge (PE) node and CE node. At a 
given UNI, more than one connection can be provisioned from the network management systems or automatically signaled from the control plane functions according to multiplexing capabilities. GMPLS controllers enable signaling of the connection establishment on demand, by communicating connectivity service end-points to the PE node. This operation can be assigned to an embedded controller to exchange the protocol messages in the form of RSVP-TE messages.

\section{Support Fixed-Mobile Convergence}

Fixed-mobile convergence means alliance of wired and wireless services and it is referring to single solutions for session control, security, QoS, charging and service provisioning for both fixed and mobile users. Fixed-mobile convergence is clearly on the roadmap of operators that want to create additional revenue streams from new value-added services.

\subsection{New Transport Networks Architectures}

Today's telecommunication networks have evolved substantially since the days of plaintelephony services. Nowadays, a wide variety of technologies are deployed, withstanding a substantial number of failures, supporting a broad range of applications based on diversified edge-user devices; they span an enormous gamut of bit-rates, and they are scaling to a large number of nodes.

In parallel, new services and networking modes (e.g., peer-to-peer) are emerging and proliferating very rapidly, modifying the temporal and spatial traffic profile in rather unpredictable ways. As has been discussed in the previous sections, it is widely recognized that the existing mind-set for the transport network architecture largely fails to accommodate the new requirements. However, the bottleneck is not only on the technology front. Architectural evolution presupposes a consensus between the many providers which, quite often, is hard to reach. This situation is exacerbating interoperability issues that, potentially, negate any competitive advantage stemming from architectural innovation. Market protectionism could stall technological advances.

Nevertheless, a major rethinking on network architectures is mandatory in the quest for a cost-effective, secure, and reliable telecommunications network. The research today is pivoted around notions on how network dynamicity can be significantly enhanced, how the cost of ownership can be reduced, and how the industrial cost of network services can be decreased. The scope of this section is to present plausible scenarios for the evolution of the core and the metropolitan transport networks, taking into account the data plane as well as the control/ management planes. It is organized so as to provide snapshots of the current situation in both segments and for three discrete time plans:

- $\operatorname{short}$ term (2010)

- medium term (2012)

- long term (2020).

Figure 1.4 depicts the existing network architecture for metro/regional and core/backbone segments, which will be the starting point in the network evolution scenario. Today, the functionality requirements are dissimilar in the two network segments, leading to the adoption of different solutions, as was shown in Figure 1.3: 


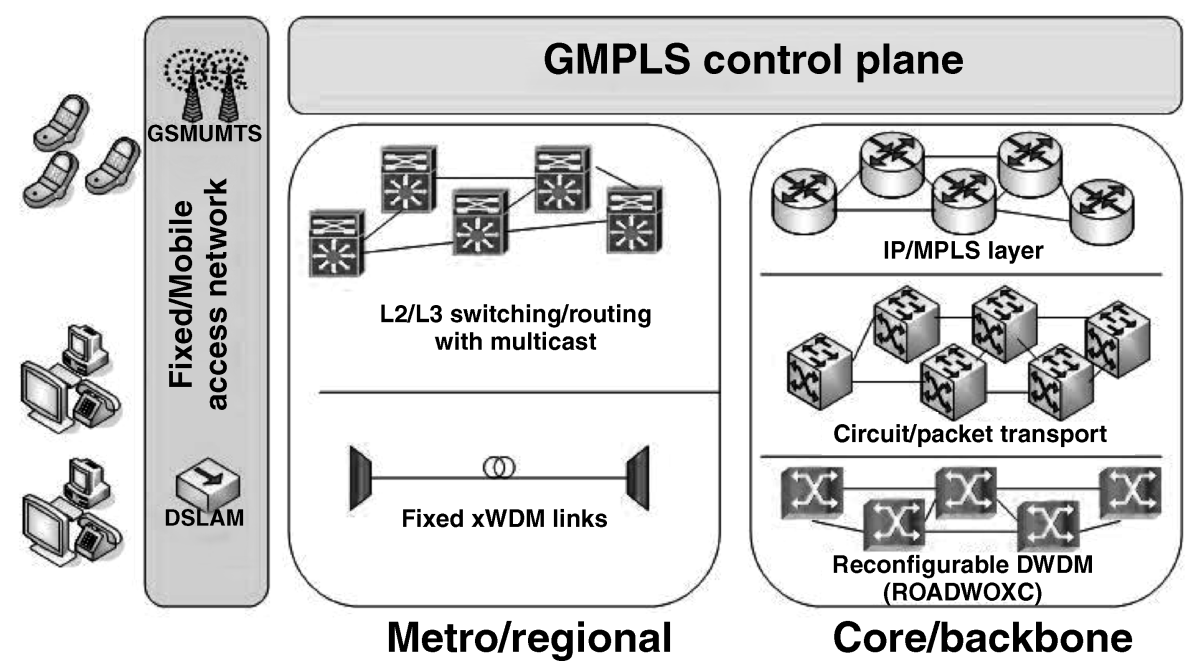

Figure 1.4 Existing metro and core network architecture

- In an MAN, the client traffic is transported from a "zoo" of protocols (IP, Ethernet, ATM, SDH/SONET, ESCON, Fiber channel, etc., to mention the most important instances only) whilst it is characterized from a low level of aggregation and grooming; this a problem exacerbated by the coexistence of unicast (video on demand, high-speed Internet and voice services) and multicast traffic (i.e., mainly IPTV). This environment postulates a highly dynamic networking, making packet-oriented solutions a necessity.

- In the core network, on the other hand, the efficient aggregation and grooming out of the MAN indicates a smoothed-out, slowly varying traffic profile, so that a circuit-switched solution is a good candidate for a cheaper switching per bit. These developments, for the core, are beefed up by the past and current developments of dense WDM (DWDM) and OTN technologies, to enhance the "bit-rate times distance" product significantly, by two to three orders of magnitude, compared to what was feasible in late 1980s, by shifting to transmission the balance for a lower cost per bit transportation.

Regarding the scenarios presented in the rest of this section, it is pointed out that their common denominator is progress in the following enablers:

- Packet technologies (in particular IP/MPLS and Ethernet) for a more efficient use of bandwidth due to the subsequent statistical multiplexing gains; that is, advanced aggregation and grooming.

- Control plane (currently dominated by ASON and GMPLS, which are further discussed in Chapter ) to decrease the cost of provisioning dramatically and the possibility to have onthe-fly resilience mechanisms.

- Optical transparency, which, as explained in Section 1.3.4.1, aims at minimizing the level of bit-by-bit processing, simplifying client signal encapsulation, leading to transparent 
bit-mapping in the transport frame and providing optical bypassing for the transit traffic. These are key functions for a reduction in CAPEX and OPEX.

As it emerges from simple inspection of the existing network architecture paradigm, efficiency and robustness today are achieved from the interplay between two, rather mutually exclusive, technologies: packets (mainly IP/MPLS and Ethernet) and circuits (SDH/SONET, OTN and WDM) do coexist in transport networks with a low level of interoperability and significant functionality duplication. Apparently, for an overall optimization, it is fundamental to increase the synergy between the layers and reduce the unnecessary functionality duplication. Thus, the emerging technologies (PTTs, see Section 1.2.9), which are in the standardization process, aim at combining the best features from both circuit and packet worlds. Therefore, features like OAM and control plane and resilience mechanisms are inherited from the circuit transport network, while frame format, statistical aggregation, and QoS support are similar to the corresponding features of packet technologies. Within the standardization bodies, two main technologies are currently under discussion: PBB-TE (IEEE802.1Qay [14] based on Ethernet) and MPLS-TP (developed in the ITU-T and IETF, starting from MPLS).

\subsubsection{Metropolitan Area Network}

The introduction of triple-play applications (voice, video, and high-speed Internet) has a strong impact on Metropolitan Area Network (MAN) traffic. The advances include improvements in residential access networks (whose traffic aggregates upwards to the MAN), multimedia distribution (which is using MANs in an intensive way) from points of presence point of presence (PoPs) to the home, and finally VPNs that are used for business and residential applications. The necessity to provide multicast services (i.e., to carry IPTV) and to add/release users to multicast groups very quickly is a strong driver towards packet solutions (IP, Ethernet, ATM, ESCON, Fiber channel, etc.).

In the MAN segment, the main advantages of circuits (low cost per switched bit per second, strong OAM, and efficient resilience mechanisms) are not essential: the bandwidth at stake is not really huge and the distances of the cables interconnecting the nodes are not very long, so that the probability of failure due to fiber cut is not that high to mandate a circuit-switched level of resilience.

However, using IP over Ethernet or pure Ethernet over WDM systems (architectural examples are available in Ref. [15]) presents some problems in terms of resilience, bandwidth guarantee, and OAM, because packet technologies currently do not have efficient mechanisms to cope with such functions. For these reasons, technologies with the ambition to couple circuitlike OAM and resilience mechanisms with packet-like flexibility, dynamicity, and granularity might represent the right candidates for next-generation networks.

Both the emerging PTTs (PBB-TE and MPLS-TP) currently have a lack of multicast traffic that, at the date of writing (July 2008), is still under study.

Figure 1.5 shows the possible evolution of the architecture for networks in the MAN or regional segment. The following sections describe in depth the concepts illustrated in this picture. At the moment, the most plausible scenario is a migrations towards technologies that, from one side, assure packet granularity and, from the other side, have "circuit-like" OAM 


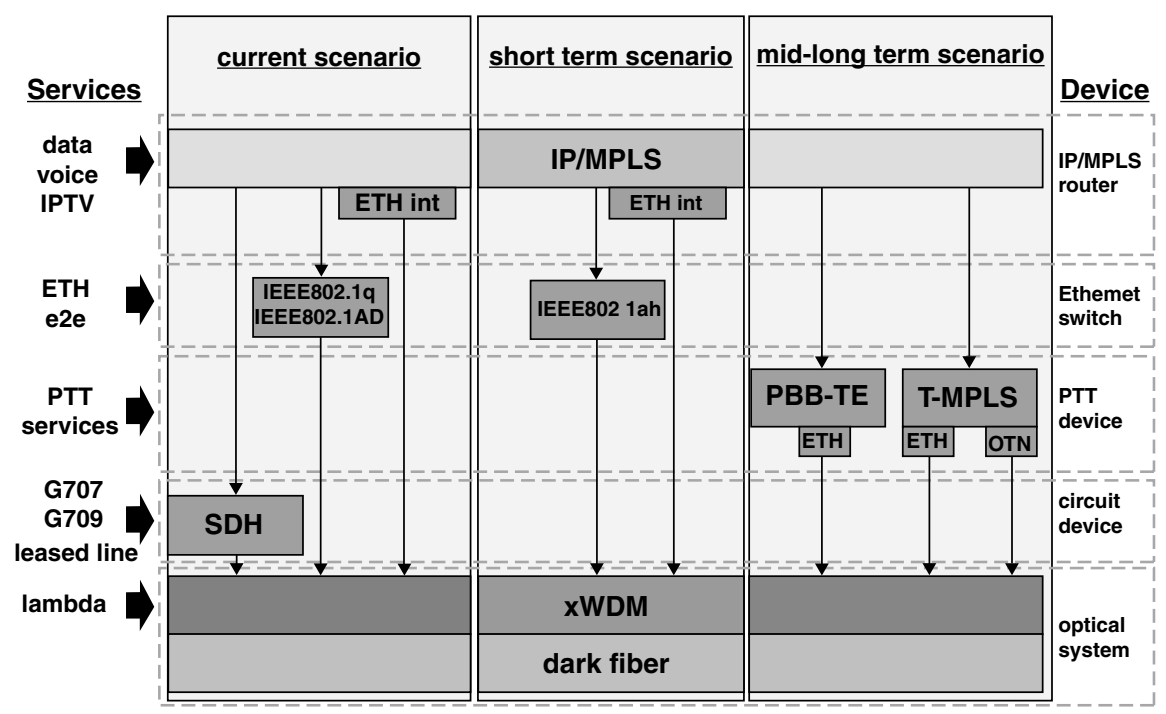

Figure 1.5 Evolution scenario for metropolitan/regional network architecture

and resilience mechanisms (capable of switching in times shorter than $50 \mathrm{~ms}$ after failure, a performance requested by the majority of applications).

\subsubsection{Short Term}

In the short term, there will probably be a progressive migration of fixed and mobile services to IP. This migration will speed up the increasing interest towards the Ethernet technology [16]. So, the roll out of native Ethernet platforms in the metro space is likely to start in the next time frame. Metro network solutions in the short term are expected to be mainly based on the Ethernet technology in star or ring topologies.

Nevertheless, in this phase, both packet (IP and Ethernet) and circuit (for the most part SDH) will coexist. In most cases, different kinds of traffic (with different quality requirements) will be carried on the appropriate platform (e.g., voice or valuable traffic) on SDH and the remainder on packet platforms.

No unified control plane is available for the transport layers. Further, the control plane is restricted to a single network domain and in most cases to a single layer within the network.

\subsubsection{Medium Term}

In the metro network, Ethernet will probably be the dominant technology in the medium-term scenario. The utilization of Ethernet is mainly driven by Ethernet conformal clients; however, non-Ethernet payloads, such as TDM, ATM, and IP/MPLS, will still exist for a long time and have to be adapted into an Ethernet MAC frame. 
Therefore, any incoming non-Ethernet payload behaves as an Ethernet payload from a network perspective; the reverse operation is performed at the outgoing interface of the egress network node.

Since Ethernet networks are growing in dimension, moving from simple LAN or basic switched networks (e.g., in a campus behavior) towards a situation where an entire metropoli$\tan$ (or regional) area is interconnected by an Ethernet platform, hundreds of thousands (or even millions) of MAC addresses would have to be learned by the switches belonging to the metro networks. To prevent this severe scalability problem, IEEE 802.1ah (PBB or MACinMAC) might be adopted. This evolution of the classical Ethernet allows layering of the Ethernet network into customer and provider domains with complete isolation among their MAC addresses.

Leaving the SDH technology, the main problems that still remain are related to the lack of efficient resilience mechanisms, present in SDH, but not mature with IP or Ethernet. In fact, traditional Ethernet (802.1q, 802.1ad, and 802.1ah) bases resilience on the "spanning tree" mechanism and its evolutions (for instance, VLAN spanning tree), which are inefficient for carrying traffic that has strong requirements in terms of unavailability.

The same argument might be argued if resilience is demanded at the IP level. In this case, routing protocols (e.g., open short path first, intermediate system to intermediate system) after a failure rearrange routing tables on surviving resources; also, this process assures stability after some seconds, a time that is often too long for voice or some video applications.

Innovative solutions to this problem might be represented by resilient packet ring (RPR) or optical circuit switching (OCS) rings. OCS rings are specially adapted to metro-core scenarios, as well as to metro access characterized by high-capacity flows between nodes (e.g., business applications and video distribution services), while RPR and dual bus optical ring network solutions fit better in scenarios with higher granularity and lower capacity requirements per access node.

At control plane level, the most important aspects that are expected for the medium-term scenario are the implementation of interfaces to make possible the exchange of information (routing and signaling) between control planes even between different domains and finally the vertical integration of the control planes of layer 1 and layer 2 technologies.

\subsubsection{Long Term}

The metro segment is composed of metro PoPs (GMPLS-capable LSR), some of which link the metropolitan network to the IP/optics core backbone (core PoP).

In this phase, the solutions based on Ethernet technology (that is, on 802.1ah (MACinMAC) and the IP/MPLS routing) will probably be replaced by innovative PTTs.

These technologies (MPLS-TP and PBB-TE) are connection-oriented transport technologies based on packet frames, enabling carrier-class OAM and fast protection. IP/MPLS should remain at the edge of the network (e.g., in the access), while the metro-core will be dominated by packet transport.

The reasons for a migration towards packet transport are a very efficient use of the bandwidth (due to the packet behavior of the connection) joint to OAM and resilience mechanisms comparable in efficiency to what is standardized in circuit-based networks. 
In addition, PTTs keep the door open to the introduction of a fully integrated ASON/GMPLS network solution, which seems to be one of the most interesting approaches to meet network emerging requirements, not only overcoming the four fundamental network problems (bandwidth, latency, packet loss, and jitter) for providing real-time multimedia applications over networks, but also enabling flexible and fast provisioning of connections, automatic discovery, multilayer TE, and multilayer resilience, all based on an overall view of the network status.

\subsubsection{Core Network}

Consistent with the metro/regional description, Figure 1.6 depicts a possible migration trend for the architecture of the backbone network.

The current network architecture, depicted in the left side of the figure, is influenced by the long-distance traffic relationships that are currently covered by two networks: an IP/MPLS network (based on routers) and a transmission network based on optical digital cross-connects (in SDH technology).

In a first phase, the evolution is represented by the migration from legacy SDH to an OTN (based on ITU-T G.709 and its evolution); in parallel, Ethernet interfaces as routers' ports will substitute PoS ports. The next phase will be characterized by the adoption of PTTs for providing connectivity and substituting pass-through routers.

The following sections describe more deeply the concepts summarized in the figure.

\subsubsection{Short Term}

In the backbone segment, for an incumbent operator, the dominance of SDH carried on DWDM systems will be confirmed in the near future.

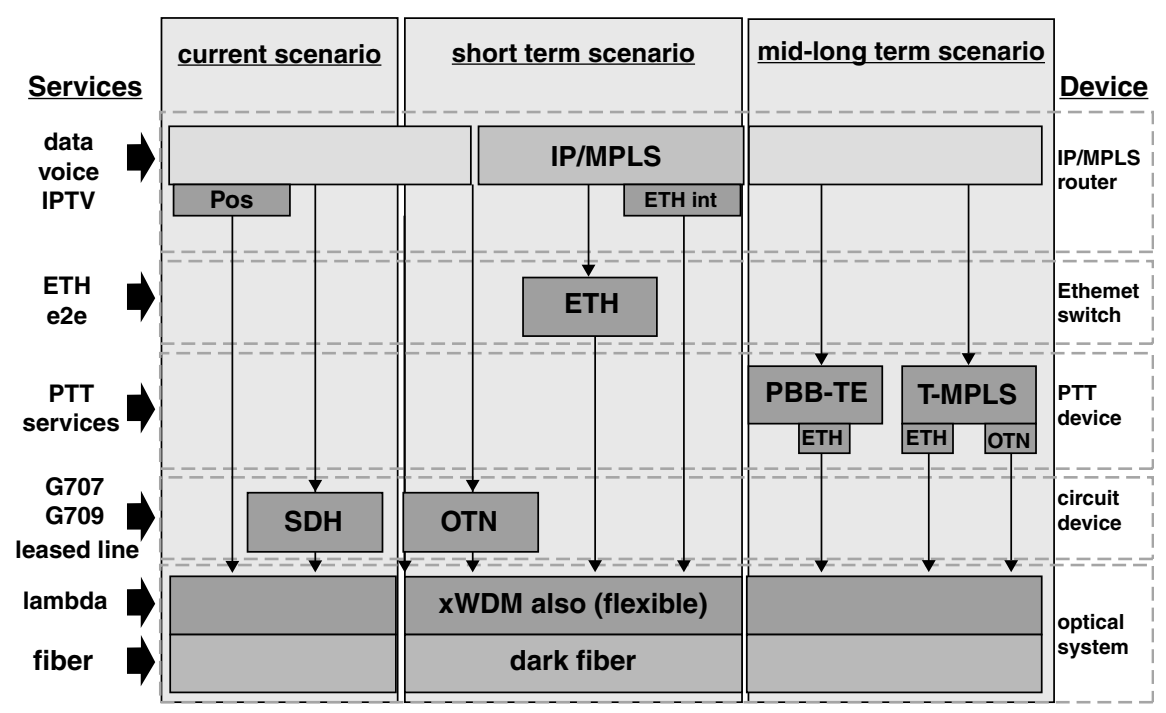

Figure 1.6 Evolution scenario for core/backbone network architecture 
No unified control plane is available for the transport layers, yet. Furthermore, the control plane is restricted to a single network domain and in most cases to a single layer within the network.

Single-layer TE and resilience mechanisms will be still in use for quite a while. The full standardization of the ASON/GMPLS control plane is not yet complete. However, some vendors already provide optical switches equipped with a standard or proprietary implementation of the GMPLS control plane and make feasible control-plane-driven networking using the overlay network model. This suggests that the automatic switched transport network architecture using GMPLS protocols is being implemented and deployed together with different releases of the UNI and NNI.

\subsubsection{Medium Term}

In the core network, standard SDH (and to some extent OTH) is being introduced. The support for Ethernet-based services is increased. The use of the native Ethernet physical layer as layer 1 transport in the core will most likely depend on the availability of OAM functionality.

With the introduction of an intelligent SDH and OTH network layer, service providers can achieve significant cost savings in their backbones. IP over static L1 networks (e.g., IP over peer-to-peer links) should cope with a high amount of transit traffic in the core routers. As traffic increases, there comes a point where the savings in IP layer expenses realized by end-to-end grooming - where the bypass traffic is sent on the L1 layer without going back to the IP layer compensate the extra expenses of introducing the intelligent layer $1(\mathrm{SDH} / \mathrm{OTH})$ switches needed.

The vertically integrated control plane refers to the underlying concepts that are called MLN/MRN at the IETF [17] and next-generation networks (NGNs) at the ITU-T. On the other hand, horizontal integration refers to the ability of control planes to provide support for service creation across multiple network domains.

The control plane will be aware of the physical layer constraints, which are important to consider, for instance, during routing in transparent/hybrid networks. Indeed, topology and resource information at wavelength level, as well as simplified signal quality/degradation information on links/wavelengths and nodes, is needed to allow the routing and wavelength assignment algorithm to place feasible paths efficiently into the network. In opaque networks, routing is based only on the overall path length constraint.

TE over domain borders between two or more domains will be a crucial topic.

\subsubsection{Long Term}

PTTs will also probably dominate the long-term scenario for the backbone segment, even if at later times than their adoption in the metro/regional network segment.

A probable architecture will consider some edge routers aggregating traffic and a network consisting of packet transport switches that will connect the edge routers.

The task of this packet transport network is to connect routers with dynamic connectivity (thanks to a control plane, probably of GMPLS type) and to assure multilayer resilience.

As shown in Figure 1.6, only some relationships might be confined at packet transport level, not the whole traffic. 
For CAPEX reasons, the deployment of packet transport devices is more similar to that of an L2 switch than to L3 routers; as a consequence, the cost of switching (normalized per bits per second) is expected to be much lower than the current cost of switching in IP routers. For this reason, large bandwidth relationships (say, larger than $2 \mathrm{Gbit} / \mathrm{s}$ ) should be carried more conveniently in connection-oriented mode. In a first phase, these circuit networks should be represented by the G.709 technology that, as said before, will probably dominate the mediumterm scenario. Successively, the architecture of the backbone network will see the coexistence of G.709 and packet transport networks.

In general, integrated equipment can be assumed for the core network in the long-term scenario; this means that, within the core network, each equipment (LSR) would integrate multiple-type switching capabilities such as packet-switching capability (PSC) and TDM (utilizing SDH/SONET or OTH fabrics); or, in an even more evolutionary scenario, a solution where PSC and lambda switching capability (LSC) or where LSC and fiber switching capability coexist may be available.

The introduction of an integrated ASON/GMPLS network control plane solution might represent one of the most interesting approaches to meet network emerging requirements, both to overcome the four fundamental network problems (bandwidth, latency, packet loss, and jitter), to provide real-time multimedia applications over networks, and to enable flexible and fast provisioning of connections, automatic discovery, multilayer TE, and multilayer resilience, all based on an overall view of the network status.

As mentioned before, the control plane model considered for the long-term scenario is a fully integrated (horizontal and vertical) GMPLS paradigm, allowing a peer-to-peer interconnection mode between network operators, as well as network domains. Specifically, full integration means that one control plane instance performs the control for all the switching capabilities present in the network.

\subsubsection{Metro and Core Network (Ultra-long-term Scenario)}

Optical burst and/or packet switching might represent important technologies to face the flexibility demand of bandwidth in future networks. It is still unclear when some implementations of these technologies will be available, even if they could be developed (both at standard level and commercially) before 2012 to 2015, which seems very unlikely.

However, a dramatic increase of the traffic amount and the necessity of end-to-end QoS, in particular for packet-based network services, may open the door to PTTs as a new layer 1/layer 2 network solution that can overcome existing shortcomings.

The further evolution of PTTs may be represented by innovative solutions based on burst/ packet switching, which would offer the following functionalities:

- Burst/packet switching will have the required dynamicity and flexibility already in layer 2, since an appropriate size of bursts/packets eliminates the grooming gap by offering a fine granularity, with less processing effort compared with short IP or Ethernet packets/frames.

- Reliability, monitoring, and QoS functionalities will be provided at layer 2, offering a solid carrier-class network service supporting higher layer network services at low cost.

- Hybrid circuit/burst/packet switching capabilities will be fully integrated into the GMPLS control plane philosophy (full vertical integration). 
Specifically for core networks, layer 2 network technologies could consist of a hybrid circuit/ burst/packet solution based on large containers carrying TDM, packet transport, and data traffic.

In metro networks - currently being dominated by Ethernet transport - the future architecture may be represented by the adoption of carrier-grade Ethernet protocols endowed with extensions on control, monitoring, and QoS. This implementation should also fit into the vertical integration strategy based on a GMPLS control plane and the horizontal integration with domain interworking providing end-to-end QoS.

From the control plane point of view in the ultra-long-term scenario, the ASON architecture based on GMPLS protocols is the most promising solution to integrate the TDM-based optical layer transport technologies (i.e., G.709) and the dominating packet-based data traffic using IP and IP over Ethernet protocols.

\subsection{Transport Networks Economics}

There is no unique infrastructure to support the required network services for the expected traffic; furthermore, not all plausible migration scenarios are cost effective for any of the network operators or within different market regulations. To analyze these differences, network operators use models that help them to evaluate how much a given network service implementation is going to cost, both in terms of CAPEX (the initial infrastructures roll out) and management and operation of the service (OPEX).

\subsubsection{Capital Expenditure Models}

CAPEX creates future benefits. CAPEX is incurred when a company spends money either to buy fixed assets or to add to the value of an existing fixed asset, with a useful life that extends beyond the taxable period (usually one financial year). In the case of telecommunications, operators buy network equipment to transmit, switch, and control/manage their infrastructures; this is part of CAPEX, but it also includes some more items:

- rights of way and civil works needed to set the equipment and deploy the lines;

- software systems (or licenses);

- buildings and furniture to house personnel and equipment;

- financial costs, including amortization and interest over loans (used to buy any of the former items).

A reasonable comparison among different solutions need not take all those items into account. However, two limiting approaches should be considered in evaluating investment and amortization:

- a "green field" situation, where a network operator starts to build their new network (or a part of it, as may happen for the access segment);

- upgrading the deployed network with new equipment or to add new functionality to the existing infrastructure. 


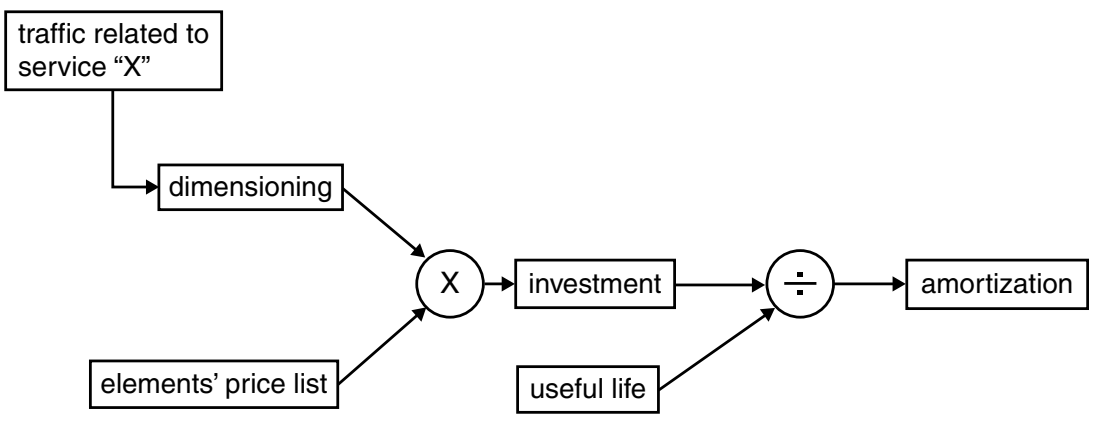

Figure 1.7 Process to evaluate the investment and amortization of a telecommunication network

As for the equipment deployment, the starting point naturally consists of dimensioning the requirements as a result of traffic estimation (Figure 1.7).

Amortization is the process of allocating one lump sum (CAPEX) to different time periods. Amortization can be calculated by different methods, but its concept describes the total expenses due to an asset over the time of its economic usefulness. As a coarse rule, the following list can be used to estimate the useful lifetime:

- network infrastructures (excavation, buildings, ...) 30 years

- optical fibers and copper wires

10 years

- network equipment

5 years

- software

3 years.

Different systems and technologies give rise to different components and, thus, different CAPEX analyses.

Figure 1.8 represents a general block model for most switching systems, including interface components, software, power supply, and common hardware elements. The cost model obviously arises from adding all individual prices for each component.

Sometimes, network operators prefer to establish a certain redundancy for some (common hardware) components so as to ensure service reliability.

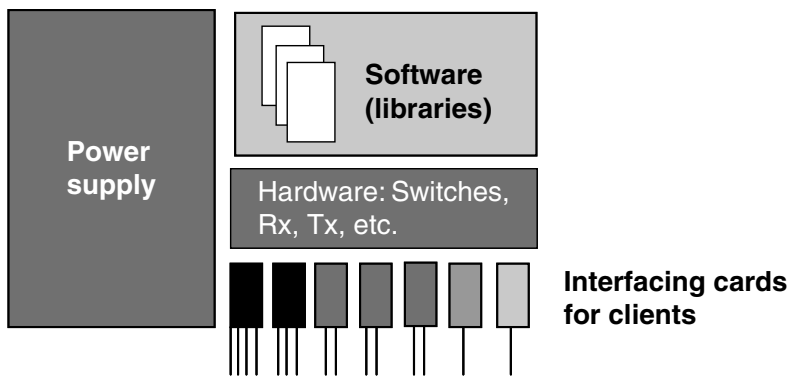

Figure 1.8 Simplified block model for a switching system 


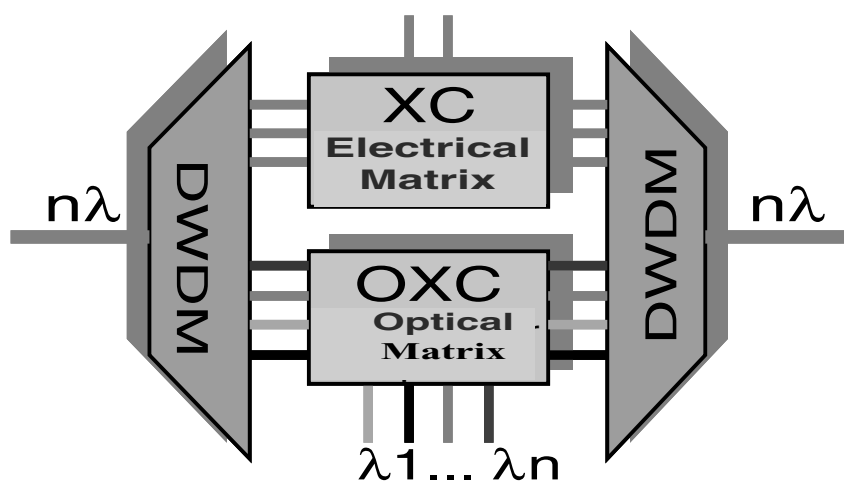

Figure 1.9 Hybrid SDH/lambda switching node

On the other hand, manufacturers also may introduce hybrid systems taking into account that common elements may be used by different technological solutions to accomplish a given function (switching packets, for instance).

Figure 1.9 shows the case for a transparent and opaque hybrid solution for a node where some lambdas are switched at the optical level, requiring no electrical regeneration (most lambdas are bypassed and transponders are only used to insert/extract lambda on/off the transmission line), whereas opaque switching (for low-granularity lines and to deal with the ingress/egress lambda of the transparent module) require electro-optical conversion. In fact, modular equipment is offered in a "pay as you grow" model to keep upgrading systems according to traffic growth for different (and interoperable) capacities like opaque/transparent hybrid nodes or L2/L3 multilevel solutions.

Aside from the amortization time schedule, network operators plan network infrastructures several years in advance. That is the reason why the introduction of new technologies must consider not only current prices, but also somewhat different ones for the future, taking into account certain conditions.

- Vendors offer discounts to network operators; discounts are very frequent because this is a way for vendors position their products as the de facto standard by massive installations.

- Equipment prices get lower after standardization agreements.

- Learning curves finally represent a significant price reduction as technologies mature. The empirical rule that states "as the total production of a given equipment doubles, the unit cost decreases in a constant percentage" can be combined with an initial estimate of market penetration to allow for predicting such a price reduction due to the maturity process.

These kinds of techno-economical prediction are usually carried out in combination with more general strategic considerations: whether network operators expect to expand their business outside present geographical limits or not, whether they will be able to reuse equipment for other purposes or places (from core networks to metropolitan ones, for example), or simply if it is possible to buy equipment from other companies, and so on.

On the other hand, cost models are always performed with a sensitivity analysis that highlights which elements are the most important to define a trend in the price evolution of 
a system and, as a consequence, to provide a tool for benchmarking it. This task must be done in combination with a general vision of the network architecture, since it is not straightforward to compare different topologies and multilevel interoperation.

\subsubsection{Operational Expenditure Models}

OPEX is not directly part of the infrastructure and, thus, is not subject to depreciation; it represents the cost of keeping the network infrastructure operational and includes costs for technical and commercial operations, administration, and so on. Personnel wages form an important part of the OPEX, in addition to rent infrastructure, its maintenance, interconnection (with other network operators' facilities) costs, power consumption, and so on.

Only considering specific OPEX derived from network services provision, the following list can be used as a guide to analyze its components:

- Costs to maintain the network in a failure-free situation. This includes current exploitation expenditures, like paying rents for infrastructures, power for cooling and systems operations, and so on.

- Operational costs to keep track of alarms and to prevent failures. This involves the main control activities to ensure QoS, namely surveying systems and their performance versus traffic behavior.

- Costs derived from failures, including not only their repair, but also economic penalties (in case an SLA states them for service failures).

- Costs for authentication, authorization, and accounting (AAA) and general management of the network.

- Planning, optimization, and continuous network upgrading, including software updating and QoS improvement.

- Commercial activities to enhance network usage, including new service offers.

Several approaches to analyzing OPEX can be used. To compare technologies and network architectures or different services implementations, a differential approach may be sufficient instead of considering all OPEX parts. However, if a business case-study requires knowledge of all costs and revenues, then a total OPEX calculation must be performed.

On the other hand, OPEX calculation is different for green-field and migration situations; for instance, bulk migration of customers or removal of old equipment will not be taken into account for a green-field scenario. Furthermore, bottom-up or top-down approaches can be used to calculate OPEX: the top-down method fits well to get a rough estimation of costs, as a starting point for a finer analysis of relative costs; the bottom-up approach is based on a detailed knowledge of operational processes.

Various approaches can be combined when dealing with OPEX calculations. In addition, OPEX and CAPEX may be balanced for accounting exploitation costs (e.g., buying or renting infrastructures) and some OPEX concepts can also be included in different items: salaries, for instance, can be considered as an independent subject or inside maintenance, commercial activities, and so on. A deep evaluation of OPEX is really important; in fact, it is possible that some technologies may offer high performances and perhaps at relative low CAPEX, but their 


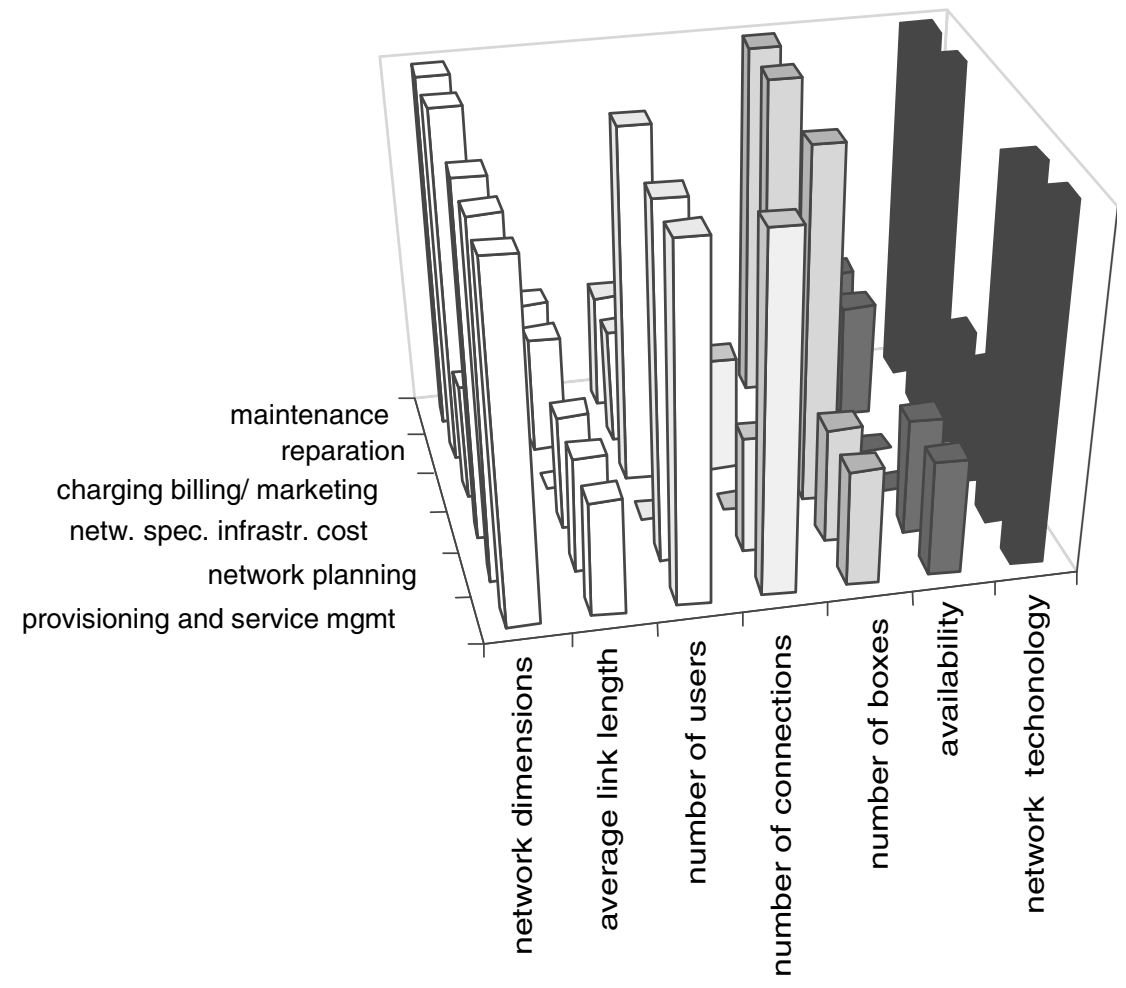

Figure 1.10 Network characteristics as cost drivers for OPEX calculations

complexity, software updating, power consumption, or personnel specialization may render them unaffordable.

Figure 1.10 shows, in arbitrary units, the dependence between OPEX components and network characteristics, considered as cost drivers so as to appreciate the impact of network technology on operational expenditure. It is clearly observed that network technologies determine the cost for maintenance, reparation, provisioning, service management, and network planning. They have less impact on charging/billing and commercial activities.

A more detailed analysis of Figure 1.10 gives the following information:

- The number of network elements (network components - e.g., routers, OXCs, PXCs) has an important impact on the cost for maintenance/reparation.

- Network technology determines not only network performance, but also some specific cost of infrastructure (more or less floor space and need of energy).

- Network dimension is important for all considered OPEX subparts, except AAA (run in centralized scheme) and marketing.

- The number of connections strongly influences the cost for provisioning and service operation and management (each connection needs to be set up), but it is less important for network planning. 
- The number of users determines the cost for provisioning, network planning, charging/ billing, and marketing, but has a small impact on the cost of maintenance and reparation.

- The average link length has little impact on maintenance, but may be significant for reparation cost when failures require a technician to go on site.

\subsubsection{New Business Opportunities}

\subsubsection{The Business Template}

Techno-economic drivers must let business progress, since network services are no longer of public strategic interest, covered by national monopolies. Such a situation is not new, but it is still evolving in accordance with clients' demands and technical improvements. Just to complete the vision and help in understanding telecommunication network evolution, some ideas about market agents and their driving actions are presented here.

The technological evolution of network infrastructure leads it to becoming multifunctional. Hence, the old scheme of one network for one purpose and kind of client, in a vertical structure (see Figure 1.11), must be changed into a matrix scheme of services based on a unique transport infrastructure: all tasks related to network service provisioning are no longer repeated for every telecommunication business; for instance, AAA is common for IPTV, teleconferencing, or POTS, as well as QoS assurance systems, network configuration, or customer commercial issues. This scheme of cross-management can also lead to telecommunication companies exploding into several specialized companies that cover a part of the business (for more than one single network operator, perhaps). In addition, the market agents involved in the telecommunication business, from content providers to end customers, play their role freely in a cross-relational model (see Figure 1.12), where commercial interests should prevail.

So, companies have to redesign their business model along the guidelines discussed so far: a method of doing business by which telecom companies generate revenues, in order to set strategies and assess business opportunities, create or get profit of synergies and so align business operations not only for financial benefits, but also to build a strong position in the
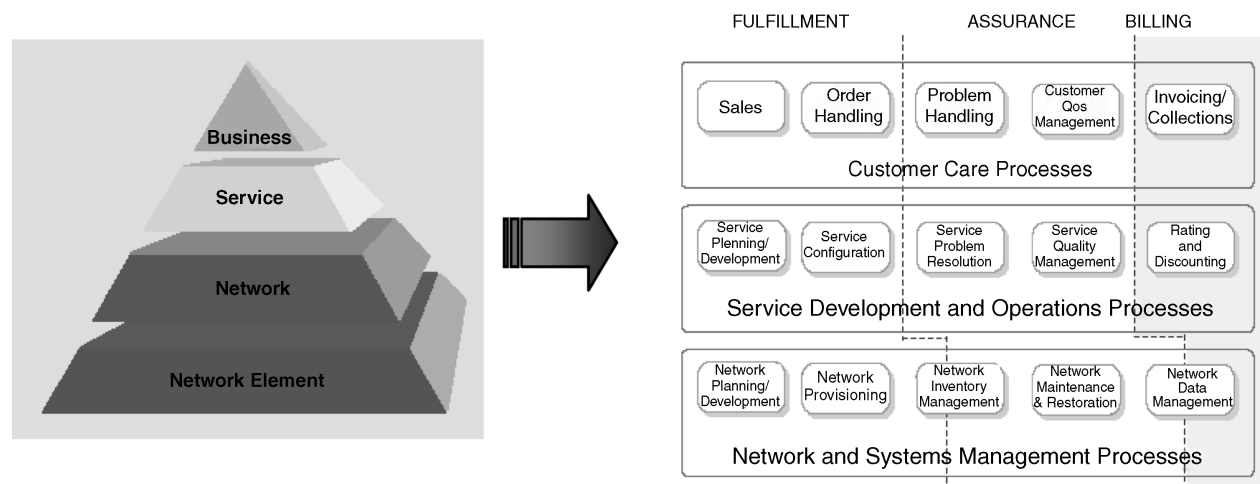

Figure 1.11 Network operator business model scheme adapted to NGN concept, from a pyramid structure to a matrix one 


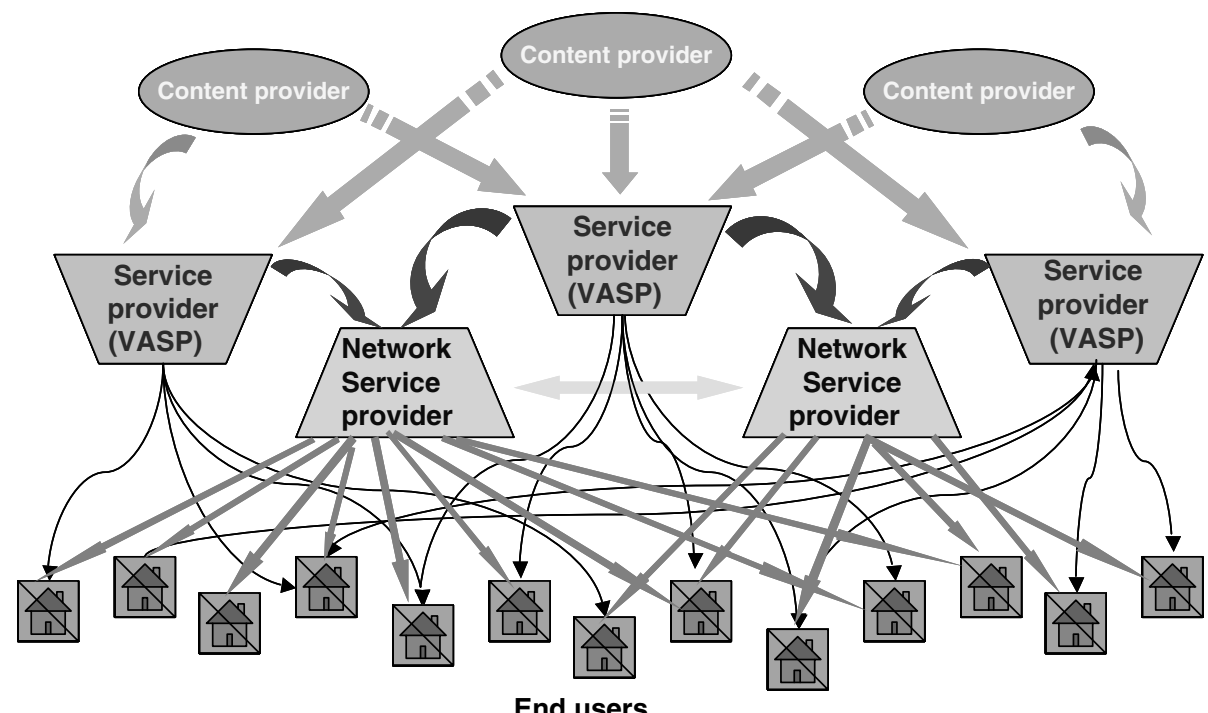

Figure 1.12 Telecommunication market agents and their cross-relationship just governed by commercial interests

market. In fact, network technology and market environment evolution affect all components of a business model, namely market segment, value proposition, value chain, revenue generation, and competitive environment. For these reasons, new business opportunities arise and the business model template has to be updated.

For the market segment, defined as the target customers group, it is clear that incumbent network operators may find virtual network operators as new clients and so the market liberalization generates new market segmentation. Each segment has different needs and expectations (e.g., business customers, banking) and companies create services for a specific types of customer; ${ }^{3}$ in addition to customer types, geographical issues must also be considered for market segmentation.

The segmentation of the market, in turn, can be based on various factors, depending on the analysis to be performed; for example, different parts of a network can be shared by different market segments; every market segment will have its own behavior, reflected in its demand model. ${ }^{4}$ Conversely, the way of using applications/services depends on the type of market segment to which they are focused (the same applications have different characteristics in residential and business areas, for example). Therefore, a proper market segmentation analysis should not only aim to map traffic demands onto transport services, but also tackle the heart of the network operator's business model: the question of how a network operator is designing

\footnotetext{
${ }^{3}$ Some broadband services target roughly the same segments, such as big enterprises (for instance, VPN services, virtual network operators, or regional licensed operators) and Internet service providers. But residential, business and public administration market segments are normally offered network services that specifically support applications for storage, grid computing, multimedia content distribution, and so on.

${ }^{4}$ This aspect includes the applications and the way they are used by those customers: frequency, time-of-the-day distribution, holding time, and so on.
} 


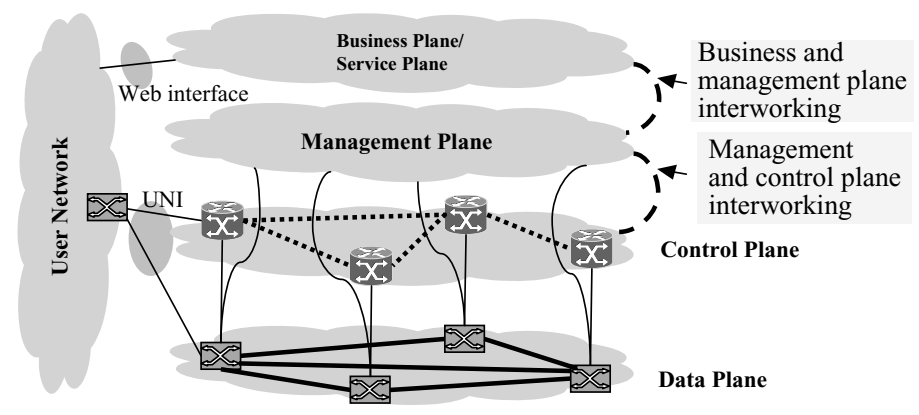

Figure 1.13 Interfaces user-network provider that may generate value proposition to different market segments

its access segment, which kind of alliance should be formed between network operators and content providers or virtual access services platform (VASP) taking into account traffic models derived from new customer demands like IPTV or new customer approaches to telecommunication issues like Web2.0. Finally, as far as network operators contract SLAs with their clients (availability and QoS), their profit depends on sharing their network infrastructure optimally.

The importance of properly segmenting the target market is critical in the development of a value proposition. The value proposition is identified by the combination of the customer needs, the services required to fulfill their needs, and the virtual value of the product. ${ }^{5}$ NGNs allow network operators to design new products/services that can have value to some customers, like those derived from VPNs and temporary bandwidth leasing (BoD). In addition, customers are going to be able to access network services using new service interfaces and procedures. These interfaces can be provided on different planes (or any combination of them):

- Service plane. VASP may offer their products with (or without) a middleware to their customers using this interface to access network resources, either via previous reservation or in a dial-in mode (with a certain service availability).

- Management plane. This is the old way. Carriers establish SLAs and reserve network resources either for dedicated connections or by statistical knowledge of traffic. Internally, management/control plane interworking functions get the transport system (data plane) and set the required connections.

- Control plane. Network operators let some users directly enter into their network control system through the UNI. In this way (as established in an SLA), those customers can set up, modify, and tear down their own VPN once the required AAA process has been performed (by the management plane).

Thus, network operators' migration activities should take into account that deployment of new services and functionalities may attract new users and increase the operator position in the market. Such an approach is worth it even if the net cash balance remains unaffected due to extra costs for implementing the new services.

\footnotetext{
${ }^{5}$ The real value of the product is formed once customers select the service among available alternatives, according to its functionality and price.
} 


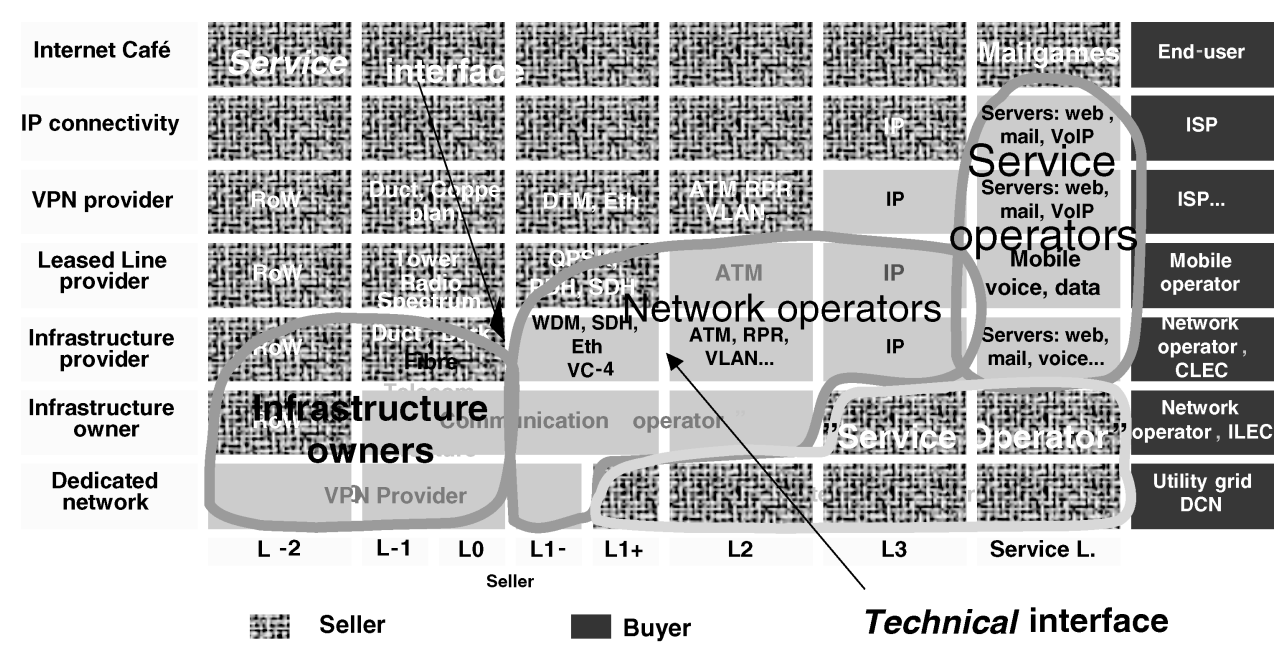

Figure 1.14 Market agents' roles mapped to the value chain by network layers to show service interfaces

On the other hand, virtual network operators (VNOs) may find easier ways of creating specialized services (TV distribution, for instance) without any handicap derived from the necessity of maintaining old services, QoS systems, or complex AAA mechanisms: their value proposition is clearly identified by a market segment. This is just the opposite case of incumbent telecommunication companies, composed of a number of departments that offer differently valued proposition services; then, synergies, market position, and customer fidelity are their main assets.

The creation of new services also modifies the value chain that represents a virtual description of the environment in which the market agents offer their services. If market desegregation implies unbundling the value chain to exploit a set of cross-relationships (Figure 1.14); also, tradeoffs and vertical alliances can be formed. These alliances are interesting whenever they produce added value to end customers because of technical improvement or service enhancement (Figure 1.14).

In fact, each box of the value chain represents an activity (Figure 1.15), and groups of activities are generally covered by independent market agents. ${ }^{6}$ This value chain scheme illustrates the connections between the telecom network layers and the corresponding telecom services; layer handovers can be commercial transactions between different organizations or enterprises, or they can be internal (technical) interfaces without any real commercial transaction. For example, there may be fiber availability issues (with underlying ducts and rights of way); the access to this dark fiber is then a service (something to sell), and the seller is known as a "dark fiber provider." The organization that is purchasing this service - the buyerhas also to place the necessary equipment infrastructure (WDM, SDH) in order to become a Network Operator. ${ }^{7}$ Other buyer-seller market profiles can be identified through the value chain in similar ways.

\footnotetext{
${ }^{6}$ Here is a key to the identification of some roles: customer, packager, connectivity provider, access network provider, loop provider, network service provider, application service provider, and content provider.

${ }^{7}$ The light boxes denote the seller and the dark boxes the buyer of the service at that interface.
} 


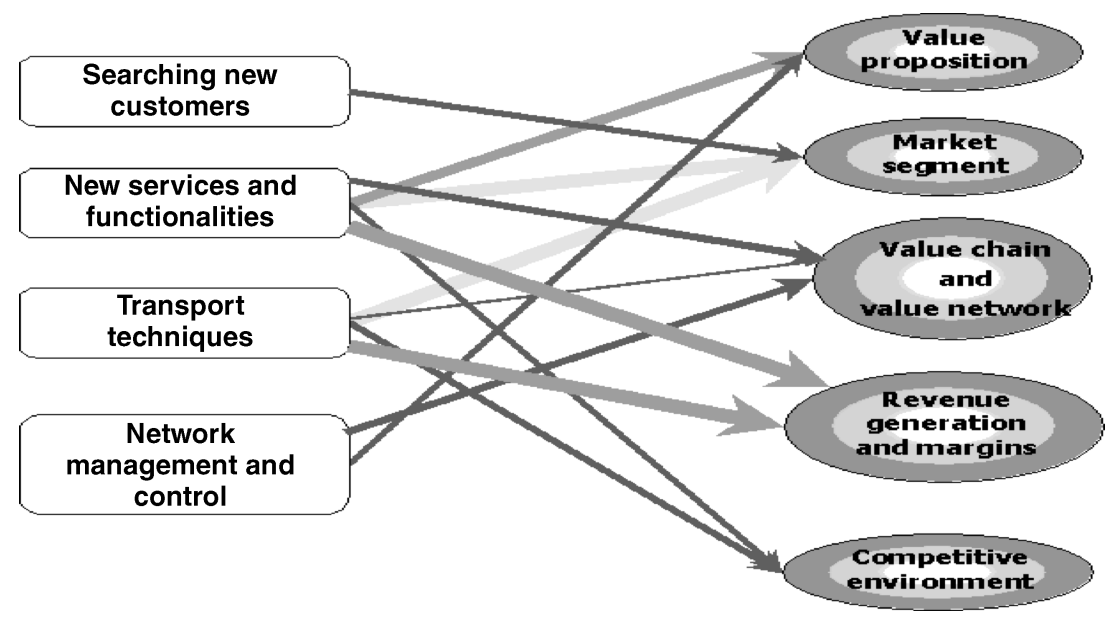

Figure 1.15 Impact of migration factors to business model components

In addition, the initial roles of the market agents can evolve according to commercial interests; for example, equipment vendors can develop network management activities, since their knowledge of the systems (hardware and software) allow them easily to propose valueadded functions; in the same way, big media companies (content providers) may become service providers or, conversely, VASP may get exclusive rights on certain contents. Network operators can split old functionalities and even outsource some of them in order to concentrate their operations in either connectivity services ("bit transmission") or services of higher quality closer to the end customer (with higher margins); on the other hand, the FMC can have different consequences in the role that network operators would like to assume in the future.

The role of network operators within the value chain scheme given in Figure 1.15 is going to comprise other activities, within the value chain, apart from selling pure network services; for instance:

- Purchasing multimedia contents and distributing them throughout a nationwide platform for other (VASP) agents to deal with them.

- Leasing or buying underlying network infrastructure (right of way, network equipment, fiber) from utility companies or other vendors and renting part of it to other network operators as their network migration is carried on.

- Renting or selling buildings and complementary equipment (for cooling, for example) when they are no longer needed by new switching and transmission systems.

- Service provisioning to other VNOs.

The revenue amount is the main assessment to determine the network operator's ability to translate the value of the product into the money received. A typical revenue model is based on monthly subscription fees and costs of transactions, commissions, and services used by customers. A supplementary income can be achieved by selling or leasing a product/service to other, nonresidential companies. In each situation a network operator should consider an appropriate pricing model in order to maximize the revenue. 
Moreover, the network migration strategy will be planned by a network operator, as long as this is possible, choosing the smallest cost of investment that may lead to greater profitability. Providing new services, after an initial investment, is a risk for several years to create new revenue-generation streams. Thus, increasing these margins is always an objective for any market agent before extending its role in the value chain or capturing new market. From the network operator point of view, it is always crucial to monitor the margins, since these are constantly change, and to plan network migration and to update the business plan, accordingly. This is not a straightforward operation; some puzzling considerations are described below:

- New services typically mean more profits from higher income; however, the higher the number of services, the more complex the management of them, aside from the effort required to search new customers. Then, the higher the diversification of services for value proposition and market segment capture, the bigger the problem with accounting and operating.

- Higher network quality and closer matching between customers' demands and network capabilities mean potentially more subscribed services and higher income. More subscribers, attracted by emerging, pioneer services lead to increasing income, provided that transport, control, and management do not overload network capabilities.

- In general, however, a higher number of customers means a faster return of investment, thus allowing reductions in the prices for services; and customizing existing services allows proposing more services and making them used more frequently, thus generating higher incomes.

- In addition, higher accessibility to the network services (through geographical expansion, higher availability derived from FMC or via interconnection facilities) and higher penetration due to combined service proposals increase the number of new subscribers and help to keep their fidelity.

- On the other hand, technical modifications affecting the traffic model will have consequences on the revenue streams. For example, the volume of metropolitan network traffic will be affected by the growth of social networks; network operators and VASP should then study carefully how IP networks are going to work in coordination with L1 and L2 transport layers and also analyze the importance of developing multicast-capable transmission systems, as well as the placement of service nodes in order to avoid bottlenecks. However, sometimes it can be proven that an extra initial investment (CAPEX) will be compensated by a consequent OPEX reduction: introduction of GMPLS for broadband standard networks will surely compensate the extra provision of bandwidth for some services unless network operators are obliged to share their transport capacity or extend it for the rest of their networks by the regulatory authority.

- Incumbent network operators must finally face the challenge of designing their network migration, not only to reach more revenue generation and gain market position, but also to keep backward compatibility. This decreases the risk of the investment and allows one to perform the infrastructure modernization in reasonable time schedules with the interesting possibility of reusing equipment.

In general, a higher value of the network and a higher position on the market allows financial advantages and new revenue streams for the telecommunication core business, like leasing offices and selling auxiliary equipment, since the eventually selected winning technology has lower requirements in terms of cooling, footprint and smaller real estate for the housing of the equipment. 
Figure 1.15 summarizes the possible impact of the already analysed components on network migration factors and to NO's business model. Perhaps all factors involved in a migration strategy will have some influence on the company competitiveness, and so a migration strategy is always designed having as a goal to increase revenues and/or to gain a better position in the competitive environment. In the latter case, a network operator can deploy new services and functionalities to develop niche markets, thus attracting new customers, and achieve diversification of services so as to extend their market segment, attracting customers that prefer dealing with a unique network operator and get advantages of synergies for both network services and AAA tasks. The impact of regulatory framework on the migration strategies has, amongst other things, the following consequences from a NO point of view:

- Changes in the regulatory framework taken from the governments may either accelerate or discourage new entrant NOs, FMC or inter company (vertical) alliances.

- Vertical or horizontal alliances, even if they do not end up in one company's takeover, modify the competition environment (for suppliers and clients, respectively). On the other hand, the advancement in standardization, in contrast to exclusive vertical (in the value chain) dependencies, also modifies the competitive environment of network operators and other telecommunication market agents.

Network operator clients are also taking advantage of new network service implementations for end-to-end broadband communication, to be based on the NGN concept; thus, the competitive environment is becoming harder, as network operators' customers can trade off different network operator service proposals and use UNI to different transport infrastructures, so as to build up their own customized VPN (see Figure 1.16) or lease part of their bandwidth capacity, thus acting as VNOs.

\subsubsection{Business Opportunities}

VPN services, regardless to the association with FMC issues, seams to be the most important driver in telecommunication business plans in the short term. Currently, VPNs are mainly supporting legacy data services in circuit switched (CS) networks. However, the advances in

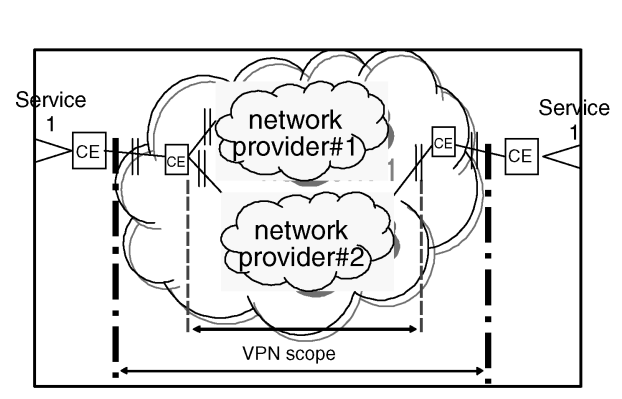

(a)

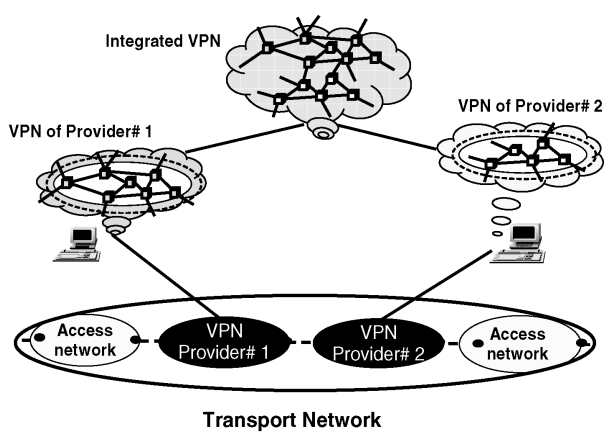

(b)

Figure 1.16 (a) Tradeoff network services from different network operators. (b) VPN concatenation to get extended services under a unified management. (CE: customer edge.) 


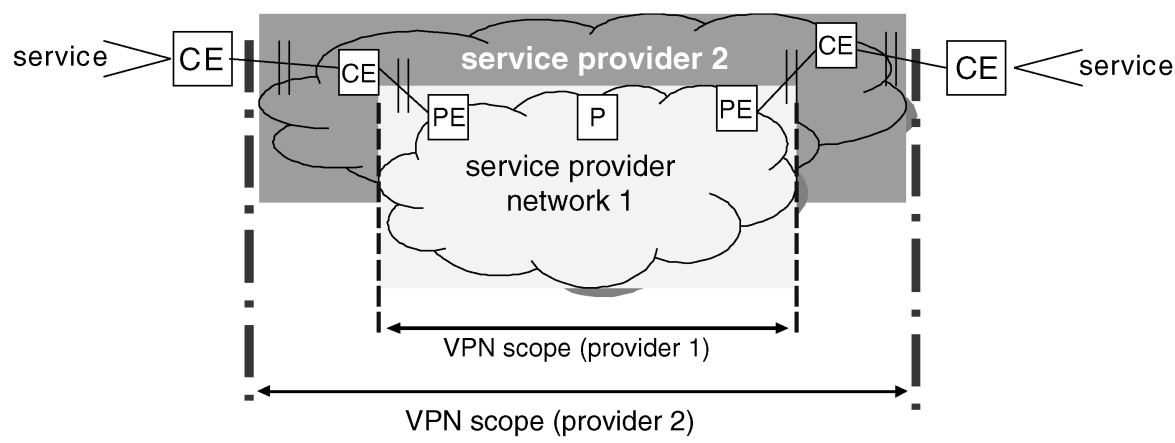

Figure 1.17 VPN cascade either to get dynamic network resources allocation or to be permanently used by VNOs (CE: customer edge; PE: provider edge; P: provider)

network technologies for VPNs are making it possible for NOs to operate a packet switched (PS) transport infrastructure like a CS one. But there is more than simulating CS networks over PS networks with the economical interest derived from statistical multiplexing and resource sharing. The currently perceived image of a "dummy" IP network will be reconsidered with the advances made possible in VPN technology: a NO may take advantage of the VPN technology to provision restricted connectivity using diffserv ${ }^{8}$-instead of over-provisioning network capacity- offering, thus, differentiated SLAs. Hence, ISPs and other VASPs could implement their preferred mechanisms to ensure application quality (for information integrity and the speed at which it reaches their customers).

A VPN may simply be used to implement VNO network resources (Figure 1.17). This fact, and the possibility of part-time network resource renting as BoD for any network operator client, is producing a radical change in the telecommunication business.

Moreover, incumbent network operators can evidently profit from operating their transport resources by means of a new developed TE approach. This policy can be based on the VPN technology, together with a network operator's ability to manage their capabilities and Ethernet network resources by reusing network elements (for the "virtuality" of the VPN paradigm) and make more scalable the management of the whole infrastructure by (virtually) dividing it and operating it as usual with the old pyramid scheme (Figure 1.17).

The expectations opened by new technologies and the market environment go beyond VPN-related topics. Improvements in network exploitation and new network service availability also give network operators new opportunities derived from:

- Cheaper and faster network upgrade (according to traffic demand) allowed by NGN. Furthermore, an NGN control plane makes it possible to adapt network resources quickly to client demands (a few minutes instead of days to reconfigure transport resources).

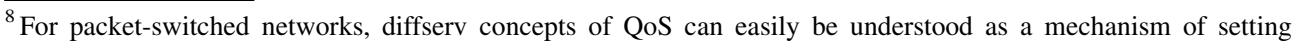
transmission preferences (at edge or core nodes) for determined sorts of packets (over a simple best-effort approach): those of a specific kind of application (real-time class, for instance) or those of a privileged customer, VPN, and so on. The concept is not new, and examples of its implementation may be found in standards of ATM services or RPR architecture. For IP networks, this non-neutral network operator performance, beyond a plain flux control, is a hot issue actively opposed by ISPs that promote ruling a net neutrality.
} 
- Reduction of time needed to provide connections and new services.

- Multicast traffic carried over p2mp connection solutions, implemented in new switching equipment, allow the introduction of specific architectures for triple-play services (namely IPTV or other multimedia applications).

- Developing Ethernet solutions for extended networks allows carriers to reduce CAPEX for wide area networks and specific-purpose networks.

- Migration from ring to mesh topologies happens to allow more efficient resilient strategies for packet-switching networks: one can think of restoration versus protection, for example.

- Complete transparent optical networks (instead of hybrid solutions) for OSI level 1 are also expected to reduce CAPEX by eliminating undue O/E/O conversions. The possibility of directly managing wavelengths also has some OPEX advantages (wavelength-switchedoriented networks).

- Parallel development of the service layer, over the control plane, is often addressed to Ethernet, new applications like storage area networks, video on demand, grid computing, and so on.

Aside from these improvements in network exploitation and in the new services arising from NGN architecture and deployment of new technologies, some collateral new business must be mentioned too, since their economical impact is not negligible.

VoIP and migration to the IPv6 protocol are making possible a complete quadruple-play offer, as well as easier ways of managing networks to provide any kind of network service, including those for real-time applications, by means of the same packet-switched-oriented equipment.

As new equipment is smaller and need less cooling and DC generators, a considerable amount of real estate is made redundant in central offices, thus allowing NOs to get extra revenues from their selling or renting. This new real estate business opportunity should be perceived as in an integral part of the current trend in extending the role of NO's towards a value added service provider which will eventually turn them to their current antithetical pole i.e. the VASP could become a (V)NO offering specialized network services. Finally, the aforementioned SLA diversification supporting dynamic network service opportunities, as well as a complex trade off between the factors affecting it, may spawn the appearance of a new market agent; that of the extended service broker. This is an agent serving as the "middle-man" between suppliers and customers, operating not only in a direct NO-client scheme but also under a dynamic and multi-step (VPN cascade, for instance) network service leasing pattern.

\section{Acronyms}

$\begin{array}{ll}\text { AAA } & \text { authorization, authentication, and accounting } \\ \text { ASON } & \text { automatic switched optical network } \\ \text { ATM } & \text { asynchronous transfer mode } \\ \text { BER } & \text { bit error rate } \\ \text { BoD } & \text { bandwidth on demand } \\ \text { CAPEX } & \text { capital expenditure } \\ \text { E-NNI } & \text { external NNI }\end{array}$


FMC fixed-mobile convergence

GMPLS generalized multi-protocol label switching

IETF Internet Engineering Task Force

I-NNI internal NNI

IPTV Internet protocol television

ITU International Telecommunications Union

LSC lambda switching capability

LSR label-switched router

MAN metropolitan area network

MEF Metro Ethernet Forum

NGN next-generation networks

NNI network-to-network interface (see ASON)

OCS optical circuit switching

O/E/O optical/electrical/optical

OIF Optical Internetworking Forum

OPEX Operational Expenditures

OSI open systems interconnection

OSS operation service support

OTH optical transport hierarchy

OTN optical transport network

OXC optical cross-connect

p2mp point-to-multipoint

PBB provider backbone bridge

PBB-TE provider backbone bridge traffic engineering

PLR packet loss rate

PoP point-of-presence

PoS packet over SONET

POTS plain old telephone service

PSC packet-switching capability

PXC Photonic Cross-connect

QoS quality of service

RPR resilient packet rings (refer to IEEE 802.17)

RSPV-TE reservation protocol with TE

SDH synchronous digital hierarchy (refer to the ITU-T framework)

SLA service level agreement

SONET synchronous optical network (refer to the ANSI framework)

TDM time-division multiplexing (see SONET and SDH)

UMTS Universal Mobile Telecommunication System

UNI user-to-network interface (see ASON)

VASP virtual access services platform

VNO virtual network operator

VoIP voice over IP

VPN virtual private network

WDM wavelength-division multiplexing

WSON wavelength-switched optical network 


\section{References}

[1] Spargins, J.D., Hammond, J., and Pawlikowski, K. (1991) Telecommunications: Protocols and Design, AddisonWesley.

[2] Dorf, R.C. (ed.) (1997) Electrical Engineering Handbook, 2nd edn, CRC Press.

[3] MEF 10.1 Technical specifications (November 2006) Ethernet services attributes Phase 2.

[4] IETF RFC 2702 (09/1999) Requirements for traffic engineering over MPLS.

[5] Davie, B. and Rekhter, Y. (2000) MPLS Technology and Applications, Morgan Kaufmann Phublishers.

[6] ITU-T Recommendation Y.1311 (03/2002) Network-based VPNs - generic architecture and service requirements.

[7] IETF RFC4026 (03/2005) Provider provisioned virtual private network (VPN) terminology.

[8] ITU-T Recommendation Y.1312 (09/2003) Layer 1 virtual private network generic requirements and architecture elements.

[9] ITU-T Recommendation Y.1313 (07/2004), Layer 1 virtual private network service and network architectures.

[10] Tomsu, P. and Wieser, G. (2002) MPLS-based VPNs, Prentice Hall.

[11] ITU-T Recommendation G.709 (03/2003) Interfaces for optical transport network (OTN).

[12] Comer, D.E. (2003) Internetworking with TCP/IP Principles, Protocols and Architectures, Prentice Hall.

[13] ITU-T Recommendations G.8080/Y.1304 (November 2001) Architecture for the automatically switched optical network (ASON).

[14] IEEE 802.1Qay (2007) Standard provider backbone bridge traffic engineering.

[15] Serrat, J. and Galis, A. (2003) IP Over WDM Networks, Artech house.

[16] Kadambi, J. Crayford, I., and Kalkunte, M. (2003) Gigabit Ethernet Migrating to High Bandwidth LANs, Prentice Hall.

[17] IETF RCF 5212 (07/2008) Requirements for GMPLS-Based Multi-Region and Multi-Layer Networks (MRN/MLN). 